\title{
6. PALEOCENE AND EOCENE DIATOM BIOSTRATIGRAPHY AND TAXONOMY OF EASTERN INDIAN OCEAN SITE 752 ${ }^{1}$
}

\author{
Elisabeth Fourtanier ${ }^{2}$
}

\begin{abstract}
The Paleocene/Eocene boundary was recovered for the first time in diatom-bearing sediments at Broken Ridge, Site 752. Diatom assemblages are documented throughout the 180-m-thick sequence of upper Paleocene to lower Eocene sediments. Age control available from magnetostratigraphy, calcareous nannofossils, and planktonic foraminifers allows calibration of diatom datum levels to absolute time. A partly new/partly revised diatom zonation is proposed for the Paleocene/early Eocene based on the results of Site 752 and consideration of other studies. The diatom zones are defined as follows (from the youngest to the oldest): Pyxilla gracilis Zone (first occurrence of Craspedodiscus undulatus to first occurrence Pyxilla gracilis); Hemiaulus incurvus Zone (first occurrence Pyxilla gracilis to first occurrence Hemiaulus incurvus); Hemiaulus peripterus Zone (first occurrence'Hemiaulus incurvus to first occurrence Hemiaulus peripterus var. peripterus). Three new taxa are described: Anaulus fennerae n. sp., Stictodiscus bipolaris n. sp., and Hemiaulus peripterus var. longispinus $\mathrm{n}$. var.
\end{abstract}

\section{INTRODUCTION}

Drilling at Broken Ridge during Ocean Drilling Program (ODP) Leg 121 resulted in the recovery of about $180 \mathrm{~m}$ of upper Paleocene and lower Eocene diatom-bearing sediments at Site 752. This paper documents the diatom assemblages and presents a zonation for the Paleocene-early Eocene based on diatom evolutionary events (first and last occurrence datums) at Site 752.

Broken Ridge started to separate from Kerguelen Plateau by seafloor spreading at around $42 \mathrm{Ma}$ (late Eocene). Since then, Broken Ridge has moved north about $20^{\circ}$ (as part of the Indo-Australian Plate), while the Kerguelen-Heard Plateau (as part of the Antarctic Plate) has had little latitudinal movement (Peirce, Weissel, et al., 1989).

A pronounced angular unconformity that crosses Broken Ridge denotes the Eocene uplift and resultant erosion following the rifting of Broken Ridge from the Kerguelen-Heard Plateau. This unconformity separates the underlying, northern-dipping Cretaceous to middle Eocene sequence from the overlying upper Eocene to Holocene cap. The dipping and truncated sequence was drilled at four sites (752-755, Fig. 1) on a 22-km-long north-south transect of holes which were chosen to allow some stratigraphic overlap between sites. The longest section of the overlying sediment cap was recovered at Site 754 with sediments ranging from upper Eocene to Holocene. Regarding the underlying sequence, middle Eocene sediments were recovered at Site 753, Maestrichtian to lower Eocene sediments at Site 752, Campanian to Maestrichtian sediments at Site 754, and Turonian to Santonian sediments at Site 755. Although drilling at these sites was done in order to provide overlapping sections, approximately $190 \mathrm{~m}$ of stratigraphic section (lower to middle Eocene) were not recovered from Sites 753 and 752, and about $460 \mathrm{~m}$ (Santonian to Campanian) were not sampled from Sites 754 and 755 (Peirce, Weissel, et al., 1989).

\footnotetext{
${ }^{1}$ Weissel, J., Peirce, J., Taylor, E., Alt, J., et al., 1991. Proc. ODP, Sci. Results, 121: College Station, TX (Ocean Drilling Program).

${ }^{2}$ Laboratoire d'Hydrologie et de Géochemie Isotopique, Université Paris-Sud, Orsay, France. (Mailing address: U.S. Geological Survey, Branch of Paleontology and Stratigraphy, MS 915, Menlo Park, CA 94025, U.S.A.)
}

Diatoms were found in calcareous sediments in the middle Eocene of Site 753 and in the upper Paleocene to lower Eocene of Site 752. Diatoms are not preserved in the sediments of Site 752 below an approximate depth of $295 \mathrm{mbsf}$; however, the presence of opal-CT in the lower Paleocene of Site 752 (Hole 752B) and in the Cretaceous of Sites 754 and 755 (Littke et al., this volume) suggests that Broken Ridge was an important site of siliceous plankton productivity in the Cretaceous through middle Eocene at Broken Ridge.

\section{Previous Studies of Paleocene and Lower Eocene Diatoms}

Our present knowledge of the stratigraphic range of Paleocene and lower Eocene diatoms is very limited because diatomaceous sequences of Paleocene and early Eocene age are rather uncommon. Figure 2 summarizes the age and location of the various sections of this age where diatoms have been studied. Diatoms have been documented from various localities of the USSR, and zonations have been proposed by Krotov and Schibkova (1961), Jousé (1979), Gleser (1978), and Strelnikova (1987). The age assignment of these diatom complexes is relatively uncertain mostly because of the lack of associated microfossils, but they appear to range from Upper Cretaceous through Eocene.

Lower Paleocene diatoms are known from deep-sea sites in the South Atlantic (ODP Sites 698 and 700: Fenner, 1991), the South Pacific (Deep Sea Drilling Project, or DSDP, Site 208: de Prado and Ling, 1981), the tropical Indian Ocean (DSDP Site 214: Jousé, 1982; Fenner, 1991), and in the North Atlantic (DSDP Site 384: Fenner, 1985b), and from terrestrial outcrops on the Seymour Island, Antarctic Peninsula (Harwood, 1988).

Upper Paleocene diatoms are known from the South Atlantic (ODP Sites 698, 699, 700, and 702: Fenner, 1991; DSDP Site 327A: Gombos, 1977; de Prado, 1981; DSDP Site 524: Gombos, 1984). They have also been documented in the South Pacific (DSDP Site 208: de Prado, 1981; de Prado and Ling, 1981), in the tropical Indian Ocean (DSDP Site 214: Jousé, 1982; Fenner, 1991; Vityaz Core 6744: Mukhina, 1974, 1976), and in the North Atlantic (DSDP Sites 384 and 398D: Fenner, 1985b).

Lower Eocene diatoms are known from the Gulf of Mexico (DSDP Site 94: Fenner, 1984, 1985b), the North Atlantic (DSDP Site 390A: Gombos, 1982; Fenner, 1984, 1985b), and the Norwegian Sea (DSDP Sites 339 and 343: Dzinoridze et al., 1978). In 


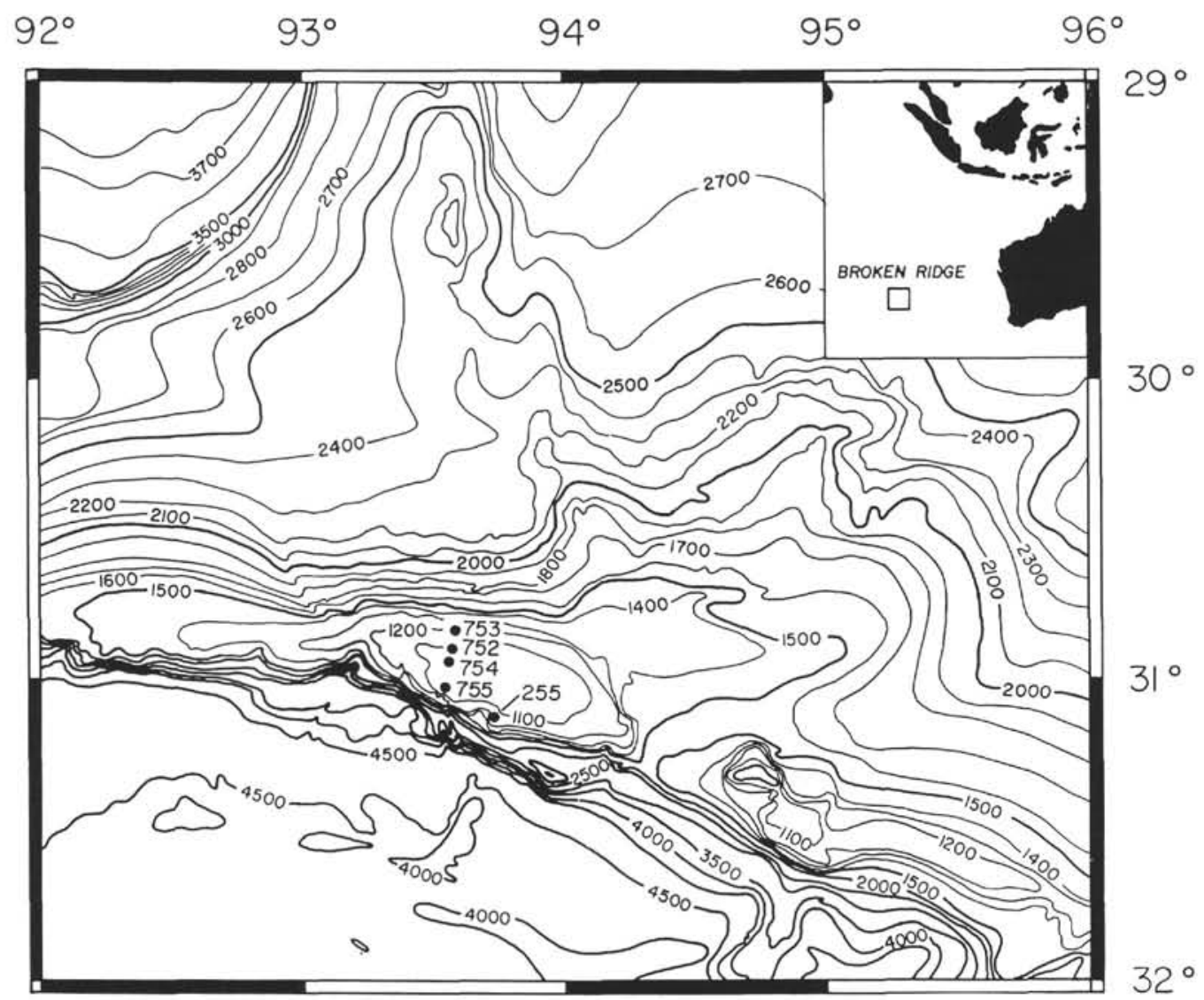

Figure 1. Location of Site 752 and other sites drilled at Broken Ridge during Leg 121.

addition to these lower Eocene deep-sea sections, diatoms are known from north Germany (Benda, 1965) and from the Fur Formation (Moler Formation) in Denmark (Benda, 1972; Fenner, 1988). Prior to drilling at Site 752, diatoms were not known in the deep-sea record neither for the earliest lowest Eocene nor at the Paleocene/Eocene boundary.

\section{Uniqueness of the Diatomaceous Section at Site 752}

Diatoms at Site 752 are of great interest because they represent the most complete and the longest section (Fig. 2) of upper Paleocene diatomaceous sediments known in the deep-sea record (about $130 \mathrm{~m}$ of sediments representing approximately $5 \mathrm{~m} . \mathrm{y}$. of the late Paleocene). Also, Site 752 is the only deep-sea section containing diatoms across the Paleocene/Eocene boundary and in the lowermost Eocene (nannofossil Zone CP9 and lowermost Zone $\mathrm{CP} 10$ ); therefore, it bridges the gap between the various deep-sea sections of late Paleocene age and those of early Eocene age.

In addition, the ages of the various diatom events are well constrained by calcareous nannofossils, planktonic foraminifers, and an excellent magnetostratigraphy (Fig. 3).

\section{METHODS}

Samples (1-3 g) were boiled with $20 \%$ hydrochloric acid (30 $\left.\mathrm{cm}^{3}\right)$ and $30 \%$ hydrogen peroxide $\left(15 \mathrm{~cm}^{3}\right)$ to remove the calcium carbonate and the organic matter. The residues prepared aboard the JOIDES Resolution (core-catcher samples) were rinsed of these chemicals by centrifuging the suspension $(75 \%$ full speed for $5 \mathrm{~min}$ ), decanting the liquid, and washing with additional distilled water (this cycle was repeated three times). The residues prepared on shore were rinsed by washing in distilled water in a
$250-\mathrm{mL}$ beaker, settling for a minimum of $8 \mathrm{hr}$, and decanting the liquid away (this cycle was repeated three or four times). Strewn slides were prepared by sampling the suspended residue with a pipette, spreading it on a $22-\times 30$-mm cover slide, drying on a hot plate, and mounting in Hyrax. At least one entire slide for each sample was scanned under the light microscope at 500x and identification of species was checked at 1250x.

The preservation of the diatom assemblages ( $\operatorname{good}=\mathrm{G}$; $\bmod$ erate $=\mathrm{M}$; poor $=\mathrm{P}$ ) was estimated based on the degree of breakage and dissolution of the valves and the relative abundance of dissolution-resistant species vs. finely silicified species. Relative abundance of the various taxa represented in the range chart is reported as abundant (A) if at least 20 specimens are present in one horizontal traverse at $500 \times$, common (C) if 19 to 3 specimens are present in each traverse, few $(F)$ if 1 or 2 specimens are present in each traverse, and rare ( $R$ ) if less than one specimen is encountered in each traverse.

\section{RESULTS}

Table 1 documents the diatom content of 43 samples examined at Site 752 (Hole 752A). Figure 3 reports the stratigraphic range of selected diatom taxa and correlates them with the magnetostratigraphy (Gee et al., this volume), the calcareous nannofossil zonation (Resiwati, this volume), and the planktonic foraminifer zonation (Van Eijden and Smit, this volume) of Hole 752A.

The diatom assemblages are usually moderately to well preserved. About 60 taxa are recorded in the checklist (Table 1); however, more taxa are present because some species (e.g., Stephanopyxis spp. and Hemiaulus spp.) are grouped together. Despite this relatively high diversity, N. I. Strelnikova (pers. 


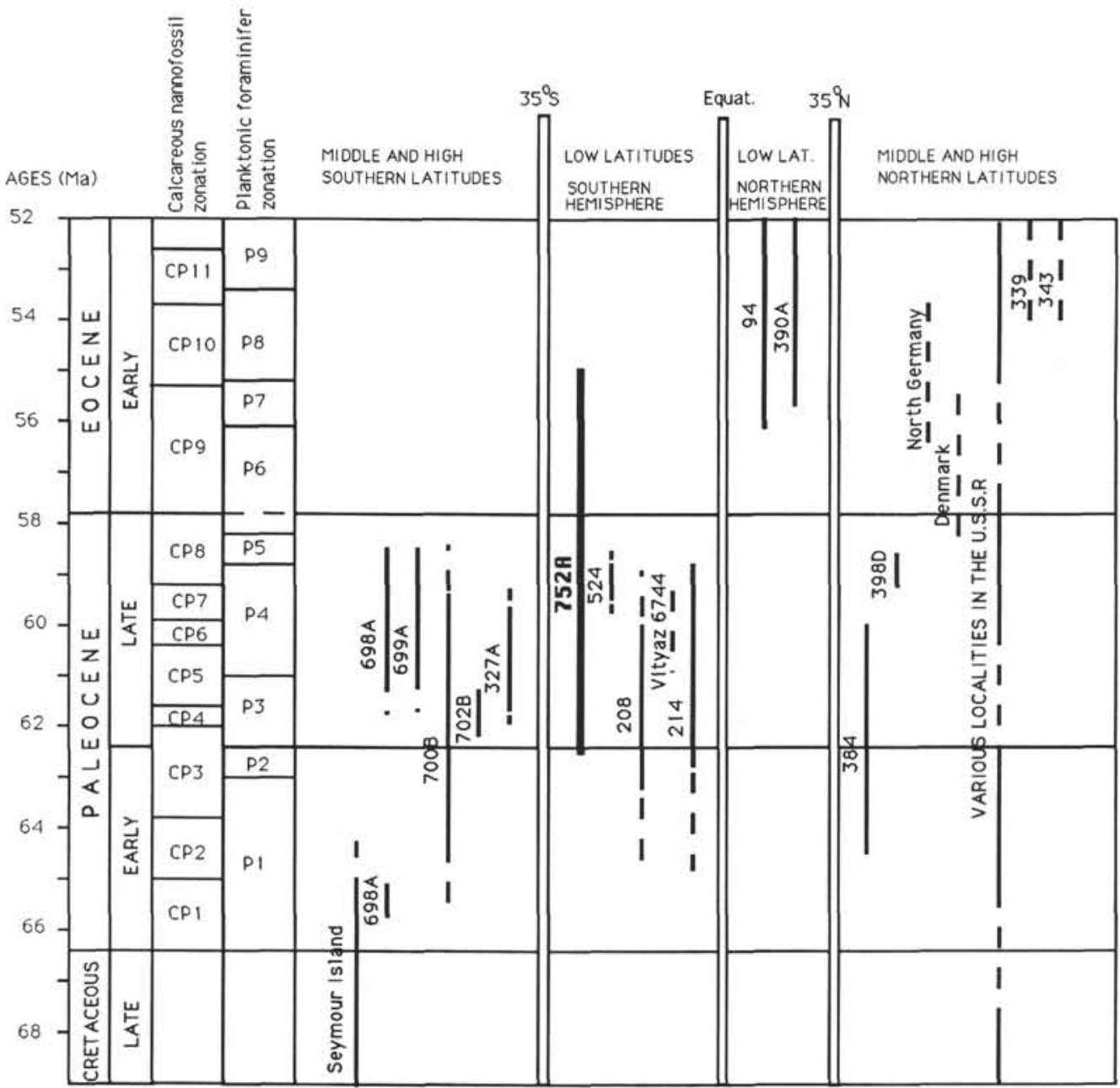

Figure 2. Ages of the various diatomaceous sections known for the Paleocene and lower Eocene. Time scale after Berggren et al. (1985a). Ages of the diatomaceous sections after compilation of calcareous nannofossil and foraminifer biostratigraphy by Fenner (1985b, 1991) for DSDP Sites 94, 208, 214, 327A, 384, 390A, 398D, and 524 and ODP Holes 698A, $699 \mathrm{~A}, 700 \mathrm{~B}$, and 702B. The sections are grouped according to geographic location: (1) middle and high southern latitudes $\left(>35^{\circ} \mathrm{S}\right)$; (2) southern low latitudes; (3) northern low latitudes; (4) northern middle and high latitudes $\left(>35^{\circ} \mathrm{N}\right)$. References for diatoms studies of the various localities (from south to north): Seymour Island-Harwood (1988); Holes 698A, 699A, 700B, and 702B Fenner (1991); DSDP Site 327A-Gombos (1977), de Prado (1981); Hole 752A — this study; DSDP Site 524 Gombos (1984); DSDP Site 208-de Prado (1981), de Prado and Ling (1981), Jousé (1982); Vityaz Core 6744 -Mukhina (1974, 1976); DSDP Site 214 -Jousé (1982), Fenner (1991); DSDP Site 94 -Fenner (1984, 1985b); DSDP 390A-Gombos (1982), Fenner (1984, 1985b); DSDP Sites 384 and 398D - Fenner (1985b); North GermanyBenda (1965); Denmark - Benda (1972), Fenner (1988); USSR - Krotov and Schibkova (1959, 1961), Sheshukova-Poretzkaja and Gleser (1964), Gleser (1978), Jousé (1979), Strelnikova (1987), etc.; DSDP Sites 339 and 343-Dzinoridze et al. (1978).

comm., 1990) indicated that the diversity of Site 752 diatom assemblages is much lower than those of the Paleocene and lower Eocene deposits of the USSR. This lower diversity may be a characteristic of oceanic deposits vs. neritic deposits. Many of the species observed at Site 752 have been previously reported from other lower Paleogene deposits of the deep-sea (e.g., Gombos, 1977, 1984; Fenner, 1991) or of mid-continent sections (e.g., Krotov and Schibkova, 1959; Jousé, 1979); however, some new taxa have been found and are described in the subsequent taxonomic section. As in other Paleocene and lower Eocene deposits, diatoms at Site 752 are mostly represented by the genera Hemiaulus, Stephanopyxis, Triceratium, and Trinacria, while the genera Coscinodiscus, Hyalodiscus, Pseudopodosira, Stellarima, and Trochosira are also well represented.

\section{Age of Selected Diatom Biostratigraphic Events at Site 752}

The age of nine diatom datum levels (Table 2) is calculated by interpolation from the sedimentation curve obtained from the depth (Peirce, Weissel, et al., 1989; Gee et al., this volume) and age (Berggren et al., 1985b) of the magnetic reversals sequence identified in Hole 752A, and on the position of the Paleocene/Eocene boundary ( $57.8 \mathrm{Ma}$, Berggren et al., 1985a) between Samples $121-752 \mathrm{~A}-19 \mathrm{X}-1,47-48 \mathrm{~cm}$, and $121-752 \mathrm{~A}-19 \mathrm{X}-3,47-48 \mathrm{~cm}$ (limit between calcareous nannofossil Zones CP8 and CP9; P. Resiwati, this volume).

The upper and lower limit of some species ranges are not very reliable at Site 752 due to sporadic occurrences (Fig. 3). However, 


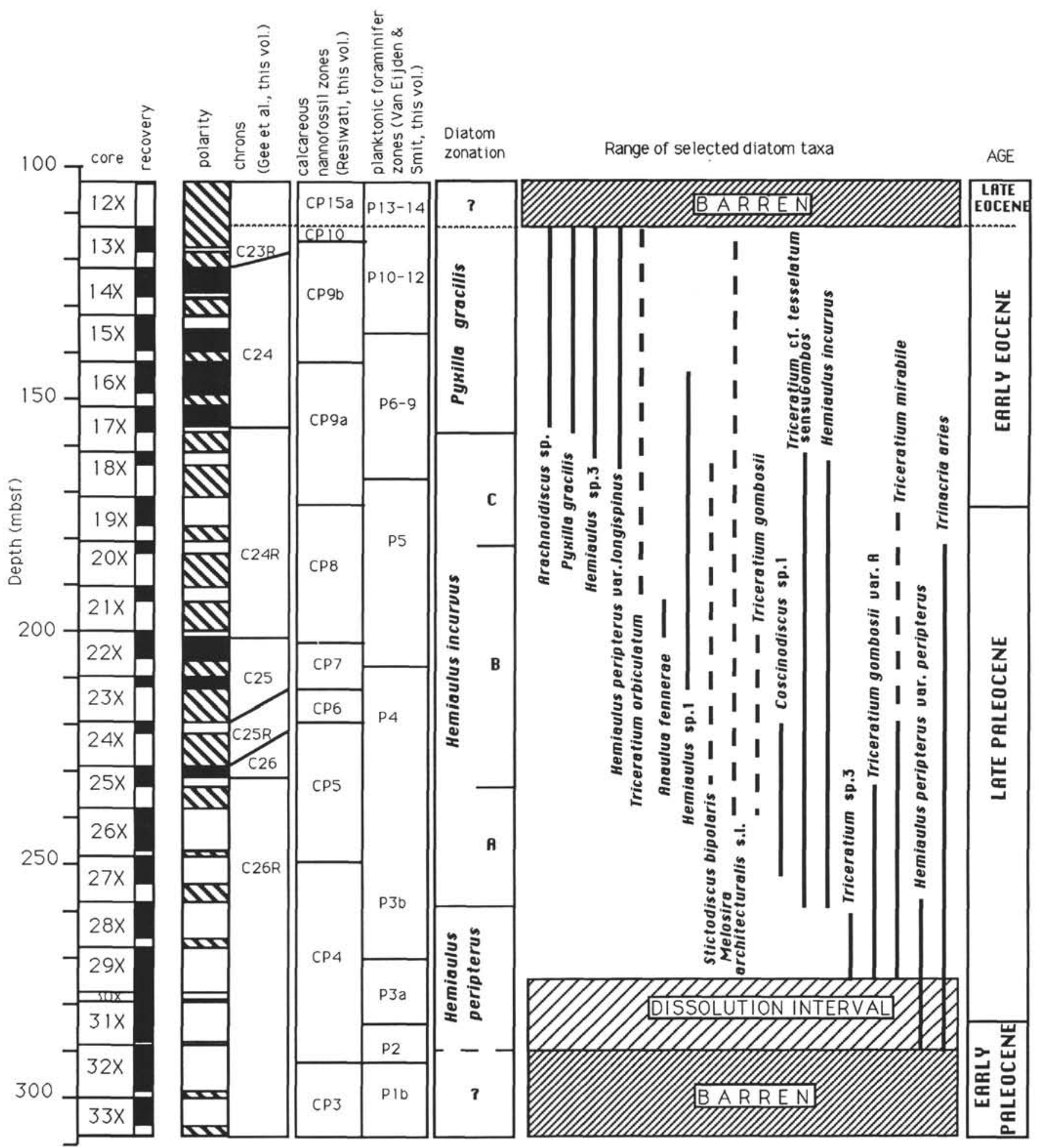

Figure 3. Range of selected diatom taxa and stratigraphy of Hole 752A. Polarity: normal polarity is indicated by black, reversed polarity is indicated by white. Diagonal ruling delineates unrecovered intervals and zones of indeterminate polarity. Ranges of diatom taxa: plain lines indicate consistent occurrence, dashed lines indicate sporadic occurrence. 
the first occurrence of Triceratium gombosii (ca. 61 Ma) is apparently reliable because it appears at a similar age (ca. $61 \mathrm{Ma}$ ) between Broken Ridge and the South Atlantic Ocean (J. Fenner, pers. comm., 1990). The other datum levels such as the first occurrences of Pyxilla gracilis, Hemiaulus peripterus var. longispinus, and Hemiaulus incurvus, and the last occurrences of Hemiaulus incurvus, Trinacria aries, Triceratium gombosii var. $\mathrm{A}$, and Hemiaulus peripterus var. peripterus are more reliable because the occurrences of these species are more consistent.

The last occurrence of Trinacria aries (57.65-57.95 Ma) approximates the Eocene/Paleocene boundary as recognized by calcareous nannofossils.

\section{Diatom Zonation for the Early Paleocene-Early Eocene Time Interval}

The only diatom zonations for the Paleocene based on the study of deep-sea sections are those of Gombos (1977, DSDP Site 327A), de Prado (1981, DSDP Sites 208 and 327A), and de Prado and Ling (1981, DSDP Site 208) (Fig. 2). These zonations could not be applied at Site 752 because they are based in part on endemic or and/or neritic diatom species not present at Site 752. Furthermore, the Gombos (1977) zonation covers only part of the upper Paleocene and the de Prado and Ling (1981) zonation does not cover the upper part of the upper Paleocene. The more complete recovery and longer section at Site 752 enabled the placement of these shorter intervals into a broader stratigraphic framework.

Various diatom zonations have been proposed for the Paleocene through Eocene based on diatom assemblages recovered in the USSR (e.g., Jousé, 1979; Strelnikova, 1987). These zones, however, are difficult to recognize in deep-sea sequences because numerous species present in the USSR deposits may be of neritic character or may have a limited geographic distribution, and are not present in deep-sea sediments. In particular, zonal markers such as Hemiaulus proteus, Coscinodiscus uralensis, and Coscinodiscus payeri, used in Strelnikova's (1987) zonation for the upper Paleocene/lower Eocene sediments of the USSR, are not found at Site 752 .

A diatom zonation, partly new and partly revised, is proposed here for the early Eocene and Paleocene based on the observation of the diatom assemblages of Site 752 (Fig. 4). Modification of Gombos's (1982) Craspedodiscus undulatus Zone is proposed in order to link Fenner's (1984) low-latitude Eocene zonation to the Eocene and Paleocene zones proposed here. In order to make this zonation usable on a larger geographic scale, the stratigraphic markers used to defined zonal boundaries are also represented in other Paleocene sections (Gombos, 1977, 1984; de Prado and Ling, 1981; Jousé, 1979, 1982; Fenner, 1991). These markers (Pyxilla gracilis, Hemiaulus incurvus, and Hemiaulus peripterus var. peripterus) are widespread, easy to recognize, and resistant to dissolution. In addition, the zones are based on first occurrence datums that are known to be generally less diachronous than last occurrence datums and cannot be reworked. Reworking does not appear to be a problem here, but this aspect may be important in applying these zones elsewhere. The subzones proposed within the Hemiaulus incurvus Zone are based on last occurrence datums and might be only of regional use.

\section{Craspedodiscus undulatus Zone}

Category. Partial-range zone.

Age. Early Eocene.

Author. Gombos (1982), modified here.

Definition. Top (modified here): first occurrence of Craspedodiscus oblongus; base (Gombos, 1982): first occurrence of Craspedodiscus undulatus.

Discussion. Gombos (1982) defined the top of the Craspedodiscus undulatus Zone by the last occurrence of Craspedodiscus undulatus; at
Sites DSDP 390A and 94 (Fenner, 1984) this event approximates the first occurrence of Craspedodiscus oblongus which is taken here as definition for the top of the zone.

Remarks. The actual first occurrence of Craspedodiscus undulatus has never been observed in the sediments. This is probably a result of the lack of overlap between lower Eocene diatoms sections at DSDP Sites $390 \mathrm{~A}$ and 94 and ODP Site 752 and/or the poor preservation of the diatoms in Cores 121-752A-13X and 121-752A-14X.

Characteristic flora. See Gombos (1982).

Correlation. According to Fenner (1985a) the first occurrence of Craspedodiscus oblongus falls into the calcareous nannofossil Zone NP13 (= Zone CP11).

\section{Pyxilla gracilis Zone}

Category. Partial-range zone.

Age. Early Eocene.

Author. Jousé (1979), modified here.

Definition. Top: first occurrence of Craspedodiscus undulatus; base: first occurrence of Pyxilla gracilis.

Secondary markers. The last occurrence of Hemiaulus incurvus approximates the base of the zone (Fig. 3).

Remarks. Jousé (1979) defined the Pyxilla gracilis Zone as an assemblage zone of early to middle Eocene age recognizable in the oceans and in the USSR. The Pyxilla gracilis Zone of Jousé (1979) is mostly characterized by the occurrence of Pyxilla gracilis and is followed in time by Jousé's (1979) Pyxilla oligocenica Assemblage Zone.

Discussion. The top of the Pyxilla gracilis Zone is not recovered in the sediments of Site 752; however, the first occurrence of Craspedodiscus undulatus is proposed here as the top of the zone in order to link the Gombos (1982) zonation for the early to middle Eocene. The first occurrence of Craspedodiscus undulatus as a definition for the top of this zone is favored because Gombos (1982) suggested a relatively large paleogeographic distribution for Craspedodiscus undulatus, whereas Pyxilla oligocenica (used in Jousé's [1979] zonation) seems to be more endemic and restricted to the high northern latitudes.

Reference locality. Site 752 (eastern Indian Ocean): Samples 121. 752A-13X-CC to $121-752 \mathrm{~A}-17 \mathrm{X}-\mathrm{CC}$.

Characteristic flora. The most characteristic taxa of this zone are Pyxilla gracilis, Triceratium orbiculatum, Arachnoidiscus sp., Hemiaulus? sp. 3, Hemiaulus peripterus var. longispinus, and Melosira architecturalis $\mathrm{s}$. ampl.

Correlation. Sediments related to this zone at Site 752 range between Cores $121-752 \mathrm{~A}-13 \mathrm{X}$ and $121-752 \mathrm{~A}-17 \mathrm{X}$ and correspond to the calcareous nannofossil Zones CP9 and lowermost CP10. The top of the zone is not recognized in Site 752. The base of the zone is located slightly above the Paleocene/Eocene boundary.

\section{Hemiaulus incurvus Zone}

Category. Partial-range zone.

Age. Late Paleocene to earliest Eocene.

Author. New zone proposed here.

Definition. Top: first occurrence of Pyxilla gracilis; base: first occurrence of Hemiaulus incurvus.

Secondary markers. The last occurrence of Hemiaulus incurvus approximates the top of this zone. The range of Triceratium $\mathrm{cf}$. tesselatum sensu Gombos (1977) is equivalent to that of Hemiaulus incurvus.

Reference locality. Site 752 (eastern Indian Ocean): Samples 121$752 \mathrm{~A}-18 \mathrm{X}-1,38-40 \mathrm{~cm}$, to $121-752 \mathrm{~A}-28 \mathrm{X}-2,100-102 \mathrm{~cm}$.

Characteristic flora. Hemiaulus incurvus, Triceratium $\mathrm{cf}$. tesselatum sensu Gombos (1977), Triceratium mirabile, and Trinacria aries are commonly found in this zone.

Correlation. At Site 752, the top of the Hemiaulus incurvus Zone correlates with the base of the calcareous nannofossil Zone CP9 and the base of the Hemiaulus incurvus Zone correlates with the top of Zone CP4.

Remarks. Hemiaulus incurvus is a diatom widespread in upper $\mathrm{Pa}$ leocene sediments. It has previously been reported in the upper Paleocene sediments of the Indian Ocean (Mukhina, 1974, 1976; Jousé, 1982) and the South Atlantic Ocean (Gombos, 1977, 1984; Fenner, 1991). It is also present in the USSR where it occurs in the upper Paleocene (N. I. Strelnikova, pers. comm., 1990). In addition, it is easy to recognize and very resistant to dissolution. The absolute age for the first occurrence of Hemiaulus incurvus in the South Atlantic of 61.3-61.9 Ma, calculated by J. Fenner (pers. comm., 1990), is similar to its estimated age of 61.60 
Table 1. Occurrence chart of diatom species in Hole 752A.

\begin{tabular}{|c|c|c|c|c|c|c|c|c|c|c|c|c|c|c|}
\hline $\begin{array}{l}\text { Core } \\
\text { Section } \\
\text { Interval (cm, top) } \\
\text { Depth (mbsf) } \\
\text { Abundance } \\
\text { Preservation }\end{array}$ & $\begin{array}{c}14 \mathrm{X} \\
1 \\
55 \\
123.15 \\
\text { C } \\
\text { PM }\end{array}$ & $\begin{array}{c}15 X \\
1 \\
55 \\
132.85 \\
\text { C } \\
P\end{array}$ & $\begin{array}{c}16 \mathrm{X} \\
3 \\
55 \\
145.55 \\
\mathrm{~A} \\
\mathrm{MG}\end{array}$ & $\begin{array}{c}17 \mathrm{X} \\
1 \\
55 \\
152.25 \\
\mathrm{~A} \\
\mathrm{MG}\end{array}$ & $\begin{array}{c}17 \mathrm{X} \\
3 \\
55 \\
155.25 \\
\mathrm{~A} \\
\mathrm{GM}\end{array}$ & $\begin{array}{c}17 X \\
4 \\
11 \\
156.31 \\
\text { C } \\
\text { MP }\end{array}$ & $\begin{array}{c}17 X \\
C C \\
156.97 \\
C \\
M\end{array}$ & $\begin{array}{c}18 \mathrm{X} \\
1 \\
38 \\
161.78 \\
\text { C } \\
\text { PM }\end{array}$ & $\begin{array}{c}18 \mathrm{X} \\
2 \\
53 \\
163.43 \\
\mathrm{C} \\
\mathrm{PM}\end{array}$ & $\begin{array}{c}18 \mathrm{X} \\
\mathrm{CC} \\
164.16 \\
\mathrm{~A} \\
\mathrm{MG}\end{array}$ & $\begin{array}{c}19 \mathrm{X} \\
1 \\
55 \\
171.65 \\
\mathrm{C} \\
\mathrm{PM}\end{array}$ & $\begin{array}{c}19 \mathrm{X} \\
3 \\
55 \\
174.65 \\
\mathrm{~A} \\
\mathrm{GM}\end{array}$ & $\begin{array}{c}20 \mathrm{X} \\
1 \\
55 \\
181.25 \\
\mathrm{~A} \\
\mathrm{M}\end{array}$ & $\begin{array}{c}21 X \\
1 \\
55 \\
190.95 \\
\text { A } \\
\text { MG }\end{array}$ \\
\hline $\begin{array}{l}\text { Actinoptychus spp. } \\
\text { Anaulus fennerae } \\
\text { Arachnoidiscus sp. } \\
\text { Coscinodiscus aff. undulosus } \\
\text { Coscinodiscus spp. }\end{array}$ & $\begin{array}{l}R \\
R \\
F\end{array}$ & $\begin{array}{c}\text { R? } \\
\text { F }\end{array}$ & $\begin{array}{l}R \\
R \\
R \\
F\end{array}$ & $\begin{array}{l}F \\
F \\
F\end{array}$ & $\begin{array}{l}R \\
F \\
F\end{array}$ & $\begin{array}{l}\mathbf{R} \\
\mathbf{R}\end{array}$ & $\begin{array}{l}R \\
R\end{array}$ & $\begin{array}{l}\mathrm{F} \\
\mathrm{C}\end{array}$ & $\begin{array}{l}\mathrm{R} \\
\mathrm{R}\end{array}$ & $\begin{array}{l}\mathrm{F} \\
\mathrm{C}\end{array}$ & R & $\begin{array}{l}\mathrm{F} \\
\mathrm{C}\end{array}$ & $\begin{array}{l}\mathrm{F} \\
\mathrm{R}\end{array}$ & $\begin{array}{l}F \\
F\end{array}$ \\
\hline $\begin{array}{l}\text { Coscinodiscus } \mathrm{sp} .1 \\
\text { Cosmiodiscus sp. } \\
\text { Fenestrella barbadensis } \\
\text { Hemiaulus inaequilateralus } \\
\text { Hemiaulus incurvus } \\
\end{array}$ & & & R? & RF & & $\mathbf{R}$ & & & R? & C & $\mathbf{F}$ & $\mathbf{R}$ & $\begin{array}{l}R \\
R\end{array}$ & $\begin{array}{c}\mathrm{R} \\
\mathrm{R} ? \\
\mathrm{C}\end{array}$ \\
\hline $\begin{array}{l}\text { Hemiaulus peripterus var. peripterus } \\
\text { Hemiaulus peripterus var. longispinus } \\
\text { Hemiaulus polycystinorum } \\
\text { Hemiaulus polymorphus var. frigida } \\
\text { Hemiaulus sp. } 1\end{array}$ & $?$ & F & $\begin{array}{l}F \\
F\end{array}$ & $\begin{array}{l}R \\
\text { C } \\
\text { C }\end{array}$ & $\begin{array}{l}\mathrm{F} \\
\mathrm{F} \\
\mathrm{F}\end{array}$ & F & $\begin{array}{l}\mathrm{F} \\
\mathrm{F}\end{array}$ & $\begin{array}{l}\mathrm{F} \\
\mathrm{F} ? \\
\mathrm{~F}\end{array}$ & $\begin{array}{l}F \\
F\end{array}$ & $\begin{array}{l}\text { F } \\
\text { C } \\
\text { F }\end{array}$ & F & $\begin{array}{l}\mathrm{C} \\
\mathrm{R} \\
\mathrm{R}\end{array}$ & $\begin{array}{c}\mathrm{C} \\
\mathrm{R} ?\end{array}$ & $\begin{array}{l}\mathrm{C} \\
\mathrm{R}\end{array}$ \\
\hline $\begin{array}{l}\text { Hemiaulus sp. } 2 \\
\text { Hemiaulus spp. } \\
\text { Hemiaulus? ciesielskii } \\
\text { Hemiaulus? conicus } \\
\text { Hemiaulus? velatus }\end{array}$ & $\begin{array}{l}\text { C } \\
\text { R }\end{array}$ & $\begin{array}{l}F \\
R\end{array}$ & c & $F$ & & $\begin{array}{l}\mathbf{R} \\
\mathrm{F}\end{array}$ & & $R$ & F & $\mathrm{F}$ & $\begin{array}{l}R \\
R\end{array}$ & $\begin{array}{l}\mathrm{F} \\
\mathrm{R} \\
\mathrm{R}\end{array}$ & $\begin{array}{l}\mathrm{F} \\
\mathrm{C} \\
\mathrm{C} \\
\mathrm{R}\end{array}$ & $\begin{array}{l}\mathrm{F} \\
\mathrm{CA} \\
\mathrm{C}\end{array}$ \\
\hline $\begin{array}{l}\text { Hemiaulus? sp. } 3 \\
\text { Hyalodiscus scoticus } \\
\text { Hyalodiscus sp. } 1 \\
\text { Hyalodiscus spp. } \\
\text { Melosira architecturalis s. ampl. }\end{array}$ & C & R & $\begin{array}{l}\mathrm{R} \\
\mathrm{R} \\
\mathrm{R} \\
\mathrm{F}\end{array}$ & $\begin{array}{l}\mathrm{F} \\
\mathrm{R} \\
\mathrm{R} \\
\mathrm{F}\end{array}$ & $\begin{array}{l}\mathrm{R} \\
\mathrm{R} \\
\mathrm{C}\end{array}$ & R & $\begin{array}{l}R \\
R \\
F\end{array}$ & $\begin{array}{l}\mathrm{R} \\
\mathrm{R}\end{array}$ & F & $\begin{array}{l}\mathrm{R} \\
\mathrm{R}\end{array}$ & & $\begin{array}{l}\mathrm{F} \\
\mathrm{F} \\
\mathrm{R}\end{array}$ & $\mathbf{R}$ & $\begin{array}{l}F \\
R \\
F \\
R\end{array}$ \\
\hline $\begin{array}{l}\text { Odontotropsis sp. } \\
\text { Paralia sulcata } \\
\text { Pseudopodosira bella } \\
\text { Pseudopodosira westii } \\
\text { Pseudopodosira sp. } 1 \\
\end{array}$ & $\begin{array}{l}\text { C } \\
\text { C }\end{array}$ & $\begin{array}{l}\mathrm{C} \\
\mathrm{F} \\
\mathrm{C}\end{array}$ & $\begin{array}{l}\mathbf{F} \\
\mathrm{C}\end{array}$ & $\begin{array}{l}F \\
F\end{array}$ & $\begin{array}{l}\text { F } \\
F\end{array}$ & F & $\mathrm{F}$ & $\mathrm{F}$ & $\begin{array}{l}R \\
R\end{array}$ & $\begin{array}{l}\mathrm{F} \\
\mathrm{F}\end{array}$ & F & $\begin{array}{l}C \\
\text { R }\end{array}$ & $\begin{array}{l}\mathrm{R} \\
\mathrm{F} \\
\mathrm{R}\end{array}$ & $\begin{array}{l}\mathrm{R} \\
\mathrm{F} \\
\mathrm{F}\end{array}$ \\
\hline $\begin{array}{l}\text { Pseudostictodiscus angulatus } \\
\text { Pterotheca spp. } \\
\text { Pyxilla gracilis } \\
\text { Rhizosolenia cretacea } \\
\text { Rhizosolenia sp. aff. } R \text {. hebetata }\end{array}$ & $\begin{array}{l}\text { C } \\
\text { C }\end{array}$ & $\begin{array}{l}\mathrm{R} \\
\mathrm{F} \\
\mathrm{F} \\
\mathrm{F}\end{array}$ & $\begin{array}{l}\mathrm{F} \\
\mathrm{A} \\
\mathrm{C}\end{array}$ & $\begin{array}{l}\text { F } \\
\text { C } \\
\text { F }\end{array}$ & $\begin{array}{l}\text { R } \\
\text { C } \\
\text { F }\end{array}$ & $\begin{array}{l}\mathrm{R} \\
\mathrm{FC} \\
\mathrm{F} \\
\mathrm{F}\end{array}$ & $\begin{array}{l}\mathrm{F} \\
\mathrm{F} \\
\mathrm{C} \\
\mathrm{R}\end{array}$ & $\begin{array}{l}\mathrm{F} \\
\mathrm{C} \\
\mathrm{R}\end{array}$ & $\begin{array}{l}\text { R } \\
\text { F } \\
\text { F }\end{array}$ & $\begin{array}{l}\mathrm{R} \\
\mathrm{F} \\
\mathrm{F}\end{array}$ & $\begin{array}{l}\mathrm{F} \\
\mathrm{F} \\
\mathrm{R}\end{array}$ & $\begin{array}{l}\mathrm{R} \\
\mathrm{C} \\
\mathrm{R}\end{array}$ & $\begin{array}{l}\mathrm{F} \\
\mathrm{C} \\
\mathrm{R}\end{array}$ & $\begin{array}{l}\mathrm{F} \\
\mathrm{C} \\
\mathrm{R}\end{array}$ \\
\hline $\begin{array}{l}\text { Sceptroneis spp. } \\
\text { Skeletonema spp. } \\
\text { Stellarima sp. } 1 \\
\text { Stellarima sp. } 2 \\
\text { Stephanopyxis spp. }\end{array}$ & C & C & $\begin{array}{l}\mathrm{F} \\
\mathrm{R} \\
\mathrm{A}\end{array}$ & $\begin{array}{l}\mathrm{F} \\
\mathrm{R} \\
\mathrm{A}\end{array}$ & $\begin{array}{l}\mathrm{F} \\
\mathrm{A}\end{array}$ & C & $\begin{array}{l}\mathrm{F} \\
\mathrm{R} \\
\mathrm{C}\end{array}$ & C & 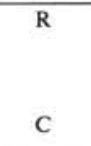 & A & C & $\begin{array}{l}\mathrm{F} \\
\mathrm{F} \\
\mathrm{F} \\
\mathrm{A}\end{array}$ & C & $\begin{array}{l}\mathrm{F} \\
\mathrm{R} \\
\mathrm{R} \\
\mathrm{C}\end{array}$ \\
\hline $\begin{array}{l}\text { Stephanopyxis aff. schulzii } \\
\text { Stictodiscus bipolaris } \\
\text { Thalassiosira? sp. 1 } \\
\text { Thalassiosiropsis sp. } \\
\text { Triceratium gombosii }\end{array}$ & & & & & & & & & R & & & & $\begin{array}{l}F \\
R\end{array}$ & R \\
\hline $\begin{array}{l}\text { Triceratium gombosii var. A } \\
\text { Triceratium mirabile } \\
\text { Triceratium aff. mirabile (coarse) } \\
\text { Triceratium orbiculatum } \\
\text { Tricer. cf. tesselatum of Gombos } 1977\end{array}$ & & & R & F & $\mathbf{R}$ & & & $\mathbf{R}$ & $\mathbf{R}$ & C & F & $\begin{array}{c}\mathrm{R} \\
\mathrm{FC}\end{array}$ & R & $\mathrm{R}$ \\
\hline $\begin{array}{l}\text { Triceratium } \text { sp. } 1 \\
\text { Triceratium } \mathrm{sp.} 3 \\
\text { Triceratium } \mathrm{spp} . \\
\text { Trinacria aries } \\
\text { Trinacria excavata }\end{array}$ & $\mathbf{R}$ & & $\mathrm{R}$ ? & C & C & C & C & A & $\mathrm{C}$ & $\begin{array}{ll}\mathrm{C} \\
\mathrm{R}\end{array}$ & $F$ & R & $\mathbf{R}$ & $\mathrm{F}$ \\
\hline $\begin{array}{l}\text { Trinacria excavata f. tetragona } \\
\text { Trinacria exsculpta } \\
\text { Trinacria pileolus } \\
\text { Trinacria simulacrum } \\
\text { Trochosira radiata } \\
\text { Trochosira spp. }\end{array}$ & & $\mathbf{R}$ & $\begin{array}{l}R \\
R\end{array}$ & $F$ & $\begin{array}{l}R \\
F\end{array}$ & $\mathbf{R}$ & $\begin{array}{l}R \\
F\end{array}$ & $R$ & $\begin{array}{l}R \\
R\end{array}$ & $\begin{array}{l}R \\
R\end{array}$ & $\mathbf{R}$ & $\begin{array}{l}\mathrm{F} \\
\mathrm{C}\end{array}$ & $\begin{array}{l}\mathrm{R} \\
\mathrm{R} \\
\mathrm{R} \\
\mathrm{R}\end{array}$ & F \\
\hline
\end{tabular}

Note: Key for symbols: $\mathrm{G}=$ good; $\mathrm{M}=$ moderate; $\mathrm{P}=$ poor; $\mathrm{A}=$ abundant; $\mathrm{C}=$ common; $\mathrm{F}=$ few; $\mathrm{R}=$ rare; $\mathrm{B}=$ barren.

61.65 Ma at Broken Ridge Site 752, and suggests a good synchroneity for this event between the Atlantic and Indian oceans.

Subzones. Three subzones (A, B, and C) are defined based on the last occurrence of Trinacria aries (boundary between Subzones C and B) and the last occurrence of Triceratium gombosii var. A (boundary between Subzones B and,A). Subzone C is defined as the interval between the top of the zone to the last occurrence of Trinacria aries. It ranges at Site 752 between Samples 121-752A-18X-1, 38-40 cm, and 121-752A-19X-3, $55-57 \mathrm{~cm}$, and is correlated to the upper portion of calcareous nannofossil Zone CP8 and to the lower portion of the CP9 Zone (earliest Eocene and latest Paleocene). Subzone B is defined as the interval between the last occurrence of Trinacria aries and the last occurrence of Triceratium gombosii var. A. It ranges at Site 752 between Samples 121-752A-20X-1, $55-57 \mathrm{~cm}$, and $121-752 \mathrm{~A}-25 \mathrm{X}-3,51-53 \mathrm{~cm}$, and correlates with calcare- 
Table 1 (continued).

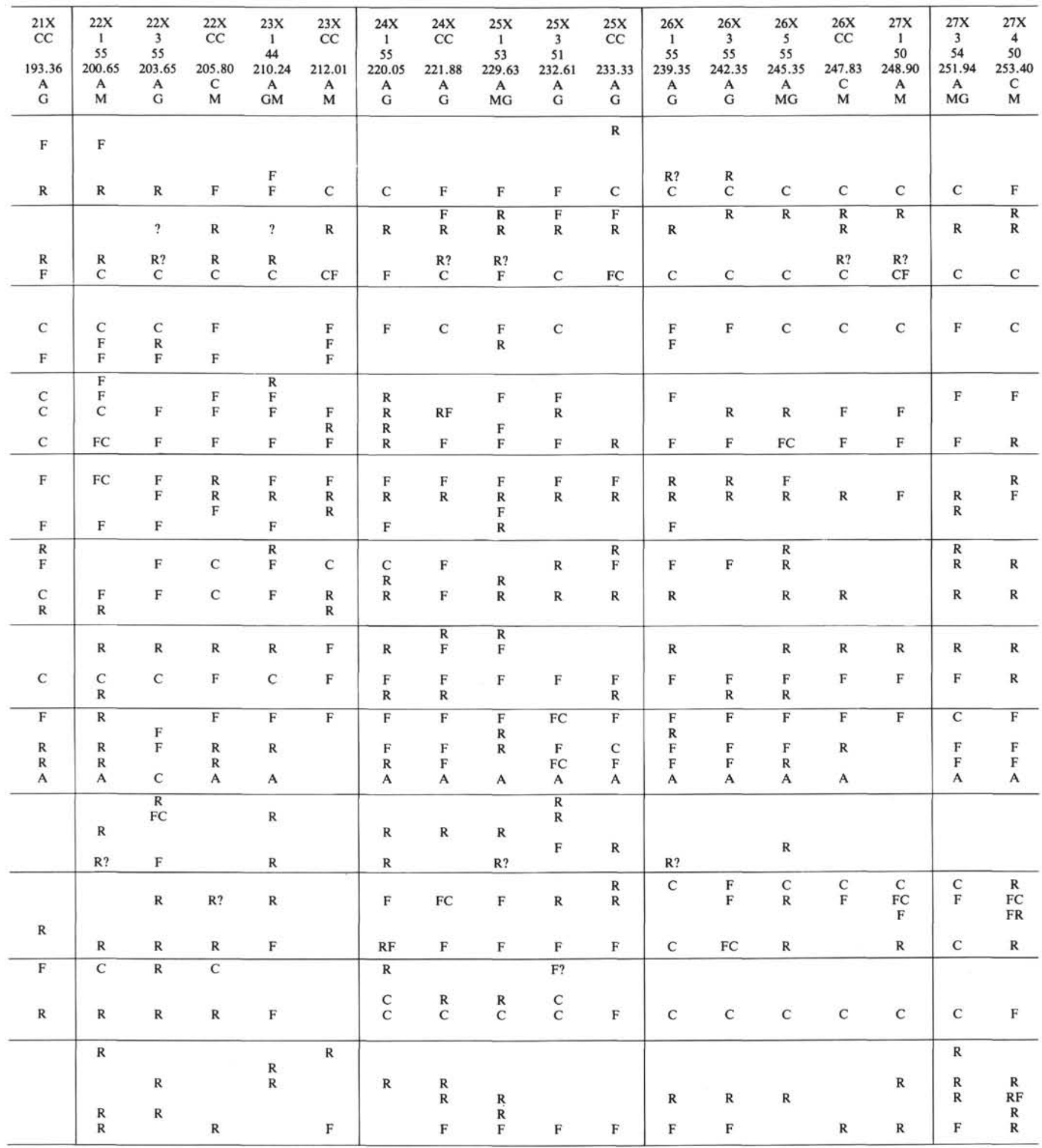




\section{E. FOURTANIER}

Table 1 (continued).

\begin{tabular}{|c|c|c|c|c|c|c|c|c|c|c|c|c|}
\hline $\begin{array}{l}\text { Core } \\
\text { Section } \\
\text { Interval (cm, top) } \\
\text { Depth (mbsf) } \\
\text { Abundance } \\
\text { Preservation }\end{array}$ & $\begin{array}{c}28 \mathrm{X} \\
1 \\
55 \\
258.65 \\
\text { F } \\
\text { MP }\end{array}$ & $\begin{array}{c}28 \mathrm{X} \\
2 \\
100 \\
260.60 \\
\text { F } \\
\text { MP }\end{array}$ & $\begin{array}{c}28 \mathrm{X} \\
3 \\
55 \\
261.65 \\
\text { C } \\
\text { MP }\end{array}$ & $\begin{array}{c}28 \mathrm{X} \\
4 \\
100 \\
263.60 \\
\text { C } \\
\text { MP }\end{array}$ & $\begin{array}{c}28 \mathrm{X} \\
5 \\
55 \\
264.65 \\
\text { FC } \\
\text { MP }\end{array}$ & $\begin{array}{c}29 \mathrm{X} \\
1 \\
55 \\
268.25 \\
\mathrm{~F} \\
\mathrm{P}\end{array}$ & $\begin{array}{c}29 \mathrm{X} \\
3 \\
55 \\
271.25 \\
\text { A } \\
\text { MG }\end{array}$ & $\begin{array}{c}29 \mathrm{X} \\
5 \\
55 \\
274.25 \\
\text { A } \\
\text { MP }\end{array}$ & $\begin{array}{c}30 \mathrm{X} \\
1 \\
54 \\
277.94 \\
\mathrm{~F} \\
\mathrm{P}\end{array}$ & $\begin{array}{c}31 X \\
1 \\
55 \\
279.95 \\
B\end{array}$ & $\begin{array}{c}31 X \\
3 \\
55 \\
282.95 \\
C \\
P\end{array}$ & $\begin{array}{c}32 \mathrm{X} \\
1 \\
55 \\
289.25 \\
\mathrm{~F} \\
\mathrm{P}\end{array}$ \\
\hline $\begin{array}{l}\text { Actinoptychus spp. } \\
\text { Anaulus fennerae } \\
\text { Arachnoidiscus sp. } \\
\text { Coscinodiscus aff. undulosus } \\
\text { Coscinodiscus spp. }\end{array}$ & C & $\mathrm{F}$ & $\mathbf{F}$ & C & R & $\mathbf{R}$ & $\mathbf{R}$ & $\mathbf{F}$ & & & $\mathrm{F}$ & $\mathbf{R}$ \\
\hline $\begin{array}{l}\text { Coscinodiscus sp. } 1 \\
\text { Cosmiodiscus } \text { sp. } \\
\text { Fenestrella barbadensis } \\
\text { Hemiaulus inaequilateralus } \\
\text { Hemiaulus incurvus } \\
\end{array}$ & $\mathrm{F}$ & $\mathrm{R}$ & $\mathbf{R}$ & & & & & & & & & \\
\hline $\begin{array}{l}\text { Hemiaulus peripterus var. peripterus } \\
\text { Hemiaulus peripterus var. longispinus } \\
\text { Hemiaulus polycystinorum } \\
\text { Hemiaulus polymorphus var. frigida } \\
\text { Hemiaulus sp. } 1\end{array}$ & $\mathbf{F}$ & $\mathrm{F}$ & C & C & C & $\begin{array}{l}\mathrm{R} \\
\mathrm{C}\end{array}$ & $\begin{array}{l}\text { F } \\
\text { C }\end{array}$ & $\begin{array}{l}\mathrm{C} \\
\mathrm{C}\end{array}$ & $\mathrm{F}$ & & RF & FR \\
\hline $\begin{array}{l}\text { Hemiaulus sp. } 2 \\
\text { Hemiaulus spp. } \\
\text { Hemiaulus? ciesielskii } \\
\text { Hemiaulus? conicus } \\
\text { Hemiaulus? velatus } \\
\end{array}$ & $\mathbf{R}$ & $\begin{array}{l}\mathrm{F} \\
\mathrm{F} \\
\end{array}$ & $\begin{array}{l}\mathrm{F} \\
\mathrm{R} \\
\mathrm{C}\end{array}$ & $\begin{array}{c}F \\
F C \\
\end{array}$ & $\begin{array}{c}\text { F } \\
\text { FC }\end{array}$ & $\mathrm{F}$ & $\begin{array}{l}\mathrm{F} \\
\mathrm{R} \\
\mathrm{F}\end{array}$ & $\begin{array}{l}\mathrm{R} \\
\mathrm{F} \\
\mathrm{F} \\
\mathrm{F}\end{array}$ & & & $\begin{array}{l}\mathbf{R} \\
\mathbf{F} \\
\mathbf{R} \\
\end{array}$ & $\mathrm{R}$ \\
\hline $\begin{array}{l}\text { Hemiaulus? sp. } 3 \\
\text { Hyalodiscus scoticus } \\
\text { Hyalodiscus } \mathrm{sp} .1 \\
\text { Hyalodiscus } \mathrm{spp} . \\
\text { Melosira architecturalis s. ampl. }\end{array}$ & & & $\begin{array}{l}\mathrm{F} \\
\mathrm{R}\end{array}$ & $\begin{array}{l}\mathrm{R} \\
\mathrm{F}\end{array}$ & $\begin{array}{l}\mathrm{R} \\
\mathrm{R}\end{array}$ & & $\begin{array}{l}F \\
R\end{array}$ & & & & & \\
\hline $\begin{array}{l}\text { Odontotropsis sp. } \\
\text { Paralia sulcata } \\
\text { Pseudopodosira bella } \\
\text { Pseudopodosira westii } \\
\text { Pseudopodosira } \mathrm{sp} .1\end{array}$ & $\mathbf{R}$ & $\mathbf{R}$ & $\begin{array}{l}\text { R } \\
\text { F }\end{array}$ & $\mathrm{R}$ & $\mathbf{R}$ & $\mathbf{R}$ & $\begin{array}{l}\mathrm{R} \\
\mathrm{R} \\
\mathrm{R}\end{array}$ & $R$ & $\mathbf{R}$ & & $\mathbf{R}$ & \\
\hline $\begin{array}{l}\text { Pseudostictodiscus angulatus } \\
\text { Pterotheca spp. } \\
\text { Pyxilla gracilis } \\
\text { Rhizosolenia cretacea } \\
\text { Rhizosolenia } \text { sp. aff. } R \text {. hebetata }\end{array}$ & $\mathrm{F}$ & & $\begin{array}{l}\mathbf{R} \\
\mathrm{R}\end{array}$ & $\begin{array}{l}\mathrm{F} \\
\mathrm{R}\end{array}$ & $\begin{array}{l}F \\
F\end{array}$ & $\mathbf{F}$ & $\begin{array}{l}\text { R } \\
\text { F }\end{array}$ & $\begin{array}{l}\mathbf{R} \\
\mathrm{R} \\
\mathrm{R}\end{array}$ & $F$ & & & $\mathbf{R}$ \\
\hline $\begin{array}{l}\text { Sceptroneis spp. } \\
\text { Skeletonema } \text { spp. } \\
\text { Stellarima } \text { sp. } 1 \\
\text { Stellarima sp. } 2 \\
\text { Stephanopyxis spp. }\end{array}$ & $\begin{array}{l}\mathrm{F} \\
\mathrm{F} \\
\mathrm{FC} \\
\mathrm{C}\end{array}$ & $\begin{array}{l}\mathrm{F} \\
\mathrm{F} \\
\mathrm{F} \\
\mathrm{C}\end{array}$ & $\begin{array}{l}\text { C } \\
\text { F } \\
\text { F } \\
\text { A }\end{array}$ & $\begin{array}{l}\mathrm{R} \\
\mathrm{F} \\
\mathrm{F} \\
\mathrm{A}\end{array}$ & $\begin{array}{l}\mathrm{F} \\
\mathrm{F} \\
\mathrm{F} \\
\mathrm{A}\end{array}$ & $\begin{array}{l}\mathrm{F} \\
\mathrm{F} \\
\mathrm{F} \\
\mathrm{A}\end{array}$ & $\begin{array}{l}\text { F } \\
\text { RF } \\
\text { F } \\
\text { A }\end{array}$ & C & $\mathrm{F}$ & & $\begin{array}{l}\text { F } \\
\text { A }\end{array}$ & $\begin{array}{l}\mathrm{R} \\
\mathrm{R} \\
\mathrm{C}\end{array}$ \\
\hline $\begin{array}{l}\text { Stephanopyxis aff. schulzii } \\
\text { Stictodiscus bipolaris } \\
\text { Thalassiosira? sp. } 1 \\
\text { Thalassiosiropsis sp. } \\
\text { Triceratium gombosii } \\
\end{array}$ & & & R & & $\mathrm{R}$ & $\mathbf{R}$ & $\begin{array}{l}\text { C } \\
?\end{array}$ & & & & & \\
\hline $\begin{array}{l}\text { Triceratium gombosii var. A } \\
\text { Triceratium mirabile } \\
\text { Triceratium aff. mirabile (coarse) } \\
\text { Triceratium orbiculatum } \\
\text { Tricer. cf. tesselatum of Gombos } 1977\end{array}$ & $\begin{array}{c}\mathrm{CF} \\
\mathrm{R} \\
\mathrm{R}\end{array}$ & $\begin{array}{l}\mathbf{R} \\
\mathbf{R}\end{array}$ & $\mathrm{R}$ ? & $\begin{array}{c}\mathrm{R} ? \\
\mathrm{R} \\
\mathrm{R}\end{array}$ & $\begin{array}{c}\mathrm{FC} \\
\mathrm{R}\end{array}$ & $\begin{array}{l}\mathbf{R} \\
\mathbf{R}\end{array}$ & $\begin{array}{l}\mathrm{F} \\
\mathrm{F}\end{array}$ & $\begin{array}{l}\mathrm{F} \\
\mathrm{R}\end{array}$ & & & R?? & \\
\hline $\begin{array}{l}\text { Triceratium sp. } 1 \\
\text { Triceratium sp. } 3 \\
\text { Triceratium spp. } \\
\text { Trinacria aries } \\
\text { Trinacria excavata } \\
\end{array}$ & $\mathbf{R}$ & $\begin{array}{l}\mathrm{R} \\
\mathrm{F}\end{array}$ & $\begin{array}{l}\text { F } \\
\text { C }\end{array}$ & $\begin{array}{c}\mathrm{C} \\
\mathrm{FC}\end{array}$ & C & $\begin{array}{c}\text { F } \\
\text { FR } \\
\text { F }\end{array}$ & $\begin{array}{c}\text { RF } \\
\text { C } \\
\text { C }\end{array}$ & $\mathrm{F}$ & $\begin{array}{l}\mathrm{R} ? \\
\mathrm{R}\end{array}$ & & $\mathbf{R}$ & $\mathbf{R}$ \\
\hline $\begin{array}{l}\text { Trinacria excavata f. tetragona } \\
\text { Trinacria exsculpta } \\
\text { Trinacria pileolus } \\
\text { Trinacria simulacrum } \\
\text { Trochosira radiata } \\
\text { Trochosira spp. }\end{array}$ & R & $\mathbf{R}$ & $\begin{array}{l}\mathbf{R} \\
\mathbf{R} \\
\mathrm{C}\end{array}$ & $\begin{array}{c}\mathrm{FR} \\
\mathrm{R} \\
\mathrm{R}\end{array}$ & $\begin{array}{l}\mathbf{R} \\
\mathrm{F}\end{array}$ & RF & $\begin{array}{l}\mathrm{F} \\
\mathrm{R} \\
\mathrm{C}\end{array}$ & F & & & $\begin{array}{l}\mathbf{R} \\
\mathbf{R}\end{array}$ & R \\
\hline
\end{tabular}


Table 2. Depth and age of diatom datum levels at Hole 752A.

\begin{tabular}{|c|c|c|c|c|}
\hline Datum & Interval & $\begin{array}{l}\text { Depth } \\
\text { (mbsf) }\end{array}$ & $\begin{array}{l}\text { Age } \\
\text { (Ma) }\end{array}$ & Chron \\
\hline & $1-752 \mathrm{~A}$. & & & \\
\hline FO Pyxilla gracilis & $17 \mathrm{X}-\mathrm{CC}$ to $-18 \mathrm{X}-1,38-40 \mathrm{~cm}$ & $156.97-161.78$ & $56.30-56.65$ & C24R (top) \\
\hline LO Hemiaulus incurvus & $18 \mathrm{X}-1,38-40 \mathrm{~cm}$, to $-18 \mathrm{X}-253-55 \mathrm{~cm}$ & $161.78-163.43$ & $56.65-56.80$ & $\mathrm{C} 24 \mathrm{R}$ \\
\hline $\begin{array}{l}\text { FO Hemiaulus peripterus } \\
\text { var. longispinus }\end{array}$ & $18 \mathrm{X}-\mathrm{CC}$ to $-19 \mathrm{X}-1,55-57 \mathrm{~cm}$ & $164.16-171.65$ & $56.85-57.40$ & $\mathrm{C} 24 \mathrm{R}$ \\
\hline LO Trinacria aries & $19 \mathrm{X}-3,55-57 \mathrm{~cm}$, to $-20 \mathrm{X}-1,55-57 \mathrm{~cm}$ & $174.65-181.25$ & $57.65-57.95$ & $\mathrm{C} 24 \mathrm{R}$ \\
\hline FO Triceratium orbiculatum & $21 \mathrm{X}-\mathrm{CC}$ to $-22 \mathrm{X}-1,55-57 \mathrm{~cm}$ & $193.36-200.65$ & $58.35-58.65$ & $\mathrm{C} 24 \mathrm{R}$ \\
\hline LO Triceratium gombosii var. A & $25 \mathrm{X}-3,51-53 \mathrm{~cm}$, to $-25 \mathrm{X}-\mathrm{CC}$ & $232.61-233.33$ & $60.75-60.80$ & C26R (top) \\
\hline FO Triceratium gombosii & $26 \mathrm{X}-1,55-57 \mathrm{~cm}$, to $-26 \mathrm{X}-3,55-57 \mathrm{~cm}$ & $239.35-242.35$ & $60.95-61.05$ & $\mathrm{C} 26 \mathrm{R}$ \\
\hline FO Hemiaulus incurvus & $28 \mathrm{X}-2,100-102 \mathrm{~cm}$, to $-28 \mathrm{X}-3,55-57 \mathrm{~cm}$ & $260.60-261.65$ & $61.60-61.65$ & $\mathrm{C} 26 \mathrm{R}$ \\
\hline $\begin{array}{l}\text { LO Hemiaulus peripterus } \\
\text { var. peripterus }\end{array}$ & $28 \mathrm{X}-5,55-57 \mathrm{~cm}$, to $-29 \mathrm{X}-1,55-57 \mathrm{~cm}$ & $264.65-268.25$ & $61.75-61.85$ & $\mathrm{C} 26 \mathrm{R}$ \\
\hline
\end{tabular}

Note: $\mathrm{FO}=$ first occurrence; $L \mathrm{O}=$ last occurrence.

AGE

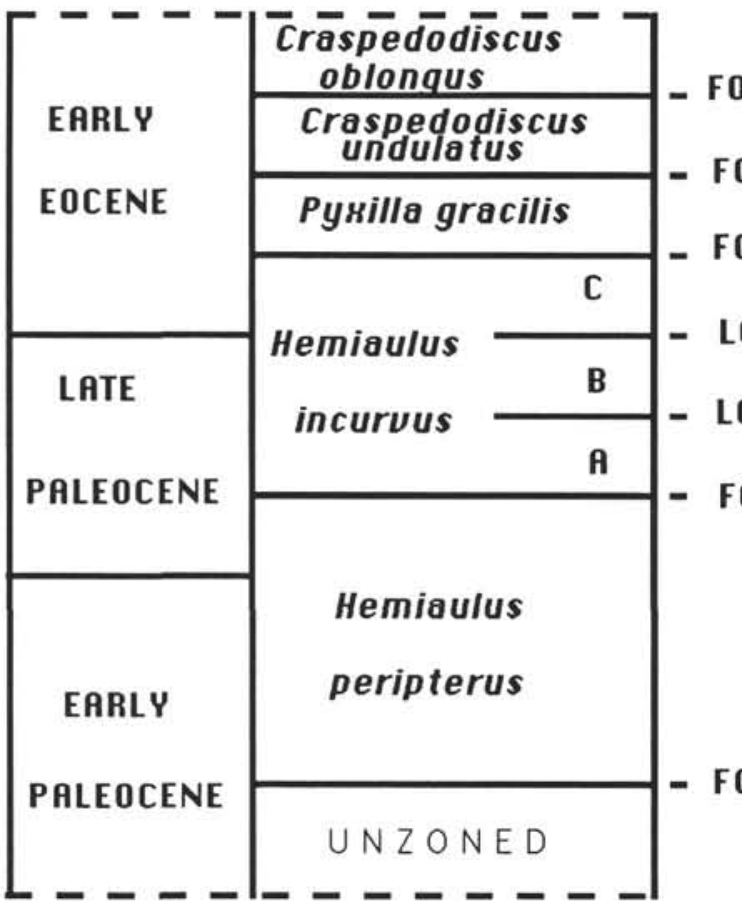

DEFINITION

Craspedodiscus oblongus

Craspedodiscus undulatus

Pysilla gracilis

Trinacria aries

Triceratium gombosii var. A

Hemiaulus incurvus

Hemiaulus peripterus

var. peripterus

Figure 4. Definition of the diatom zones for the early Eocene and Paleocene.

ous nannofossil Zones upper CP5, CP6, CP7, and lower CP8 (latest Paleocene). Subzone A is defined as the interval between the last occurrence of Triceratium gombosii var. A and the first occurrence of Hemiaulus incurvus. It ranges at Site 752 between Samples 121-752A-25X-CC and $121-752 \mathrm{~A}-28 \mathrm{X}-2,100-101 \mathrm{~cm}$, and correlates with the upper portion of the calcareous nannofossil Zone CP5 and the lower portion of the CP4 Zone (middle late Paleocene).

\section{Hemiaulus peripterus Zone}

Category. Partial-range zone.

Age. Early Paleocene to late Paleocene.

Author. New zone proposed here.

Definition. Top: first occurrence of Hemiaulus incurvus; base: first occurrence of Hemiaulus peripterus var. peripterus.

Secondary markers. At Site 752, the last occurrence of Hemiaulus peripterus var. peripterus approximates the top of the zone (Fig. 3).
Reference locality. Site 752 (eastern Indian Ocean): Samples 121 $752 \mathrm{~A}-28 \mathrm{X}-3,55-57 \mathrm{~cm}$, to $121-752 \mathrm{~A}-32 \mathrm{X}-1,55-57 \mathrm{~cm}$.

Remarks. de Prado and Ling (1981) defined the Hemiaulus caracteristicus Zone from the lower Paleocene of DSDP Site 208 between the first occurrence of Hemiaulus caracteristicus s. ampl. and the first occurrence of Notiostyrax tasmanos. Examination of slides from this site indicates that Hemiaulus caracteristicus s. ampl. documented by de Prado and Ling (1981) between Cores 29 and 31 of DSDP Site 208 is the synonym of Hemiaulus peripterus var. peripterus. Photographs of this taxon in de Prado (1981) are also of Hemiaulus peripterus var. peripterus (D. Harwood, pers. comm., 1990).

Discussion. The first occurrence of Hemiaulus peripterus var. peripterus is not observed in the sediments of Site 752, as the diatoms are present only to an approximate depth of $295 \mathrm{mbsf}$ (uppermost part of the lower Paleocene), due to preservational loss. This species is known to occur in the lower and upper Paleocene (Fenner, 1991) of numerous 
sections. However, it has never been reported from the Cretaceous nor from the lowermost Paleocene (e.g., Harwood, 1988). This suggests an early Paleocene age for the first occurrence of Hemiaulus peripterus var. peripterus.

\section{CONCLUSION}

Site 752 contains the longest $(180 \mathrm{~m})$ continuous section of Paleocene to lower Eocene diatomaceous sediments in the deepsea record (representing about 8 m.y.). The ages are well constrained by calcareous microfossil stratigraphy and magnetostratigraphy, allowing accurate correlation of the diatom biostratigraphic events. Site 752 represents to date the best reference section for siliceous microfossils during the late Paleocene-early Eocene time interval. Three zones and three subzones are proposed for this interval and as many as nine stratigraphically useful diatom events (last and first occurrences) are identified.

Results presented in this paper should be useful for improving correlation of Paleocene-Eocene diatomaceous sections which may not contain calcareous microfossils. In particular, thick diatomaceous sections of Paleocene and Eocene age are known in the USSR; however, their correlation is presently uncertain because of the absence of calcareous microfossils and magnetostratigraphy in these sections (N. I. Strelnikova, pers. comm., 1990). Some of the diatom biostratigraphic events documented at Site 752 may be recognized in USSR deposits, providing a way of improving the correlation of these deposits.

\section{FLORAL REFERENCES AND TAXONOMIC NOTES}

\section{Description of New Taxa}

Hemiaulus peripterus Fenner 1984 var. longispinus Fourtanier, n. var. (PI. 3, Figs. 5, 8, and 9)

Description. Valve narrow with straight, elongate narrow apical elevations terminated with an acute spine (Pl. 3, Fig. 5) and straight, long acute downward extensions of the mantle at each pole. The valves appear mostly structureless, but some small pores can be observed on the apical elevations (Pl. 3, Fig. 9) and a small structure is present at the center of the valve. In girdle view, the valve is straight along the apical axis, and the horns and downward extension are straight and perpendicular to the apical axis.

Diagnosis. This taxon resembles Hemiaulus peripterus Fenner 1984 (pl. 1, figs. 8,9 ) by the structure of the valve. Differences between the two taxa are the shape of the valve in girdle view, which is curved for Hemiaulus peripterus var, peripterus and straight for Hemiaulus peripterus var. longispinus, and the length of the downward apical extension, which is relatively short for Hemiaulus peripterus var. peripterus and long for the variety longispinus.

Remarks. The unnamed specimen illustrated in Schmidt et al. (18741959 , pl. 142, fig, 12) looks identical to Hemiaulus peripterus var. longispinus $\mathrm{n}$. var.

Occurrence. Both Hemiaulus peripterus var. peripterus and Hemiaulus peripterus var. longispinus occur at Site 752. The two forms, however, have a different, non-overlapping stratigraphic range, which supports their separation (Fig. 3). At Site 752, Hemiaulus peripterus var. peripterus ranges in the upper lower Paleocene and in the lower upper Paleocene (Cores 121-752A-29X to 121-752A-32X) whereas Hemiaulus ranges younger, in the earliest Eocene (Cores 121-752A-16X to 121$752 \mathrm{~A}-18 \mathrm{X}$ ).

Holotype. Smear slide \#216012 deposited at the California Academy of Sciences (Pl. 3, Fig. 9)

Type locality. Eastern Indian Ocean, Sample 121-752A-17X-3, 55$57 \mathrm{~cm}$.

Age. Earliest Eocene.

\section{Anaulus fennerae Fourtanier, n. sp.}

(Pl. 4, Figs. 5-7; Pl. 5, Fig. 1)

Description. In valvar view, the valve outline appears linear, with slight inflation near the center. The apices are rounded. The valve length varies between 20 and $45 \mu \mathrm{m}$, the width between 6 and $10 \mu \mathrm{m}$. The valve is segmented by transapical septae (ca. $3-4$ in $10 \mu \mathrm{m}$ ) which are not exactly perpendicular to the apical axis. Most of the septae cross the valve but some of them are marginal only. In scanning electron microscopy (P1. 5, Fig. 1), the valve appears slightly undulated with each undulation corresponding to an internal septa. The valve surface is covered with small pores (ca. 15 in $10 \mu \mathrm{m}$ ), irregularly arranged. In girdle view (Pl. 4, Fig. 7), it is possible to observe the continuation of the septae and the delicate punctuation of the mantle. This species forms chain-colonies (P1. 4, Fig. 7).

Remarks. This species is tentatively placed in the genus Anaulus Ehrenberg. I note some structural analogies with Huttonia virgata Grove et Sturt (1887), but the absence of ocelli on my specimens prevents the assignment to Huttonia, and the placement of this species in the genus Anaulus is the most likely choice at this time.

Occurrence. Anaulus fennerae has a short range in the upper Paleocene at Site 752 and occurs in significant numbers in Cores 121-752A$21 \mathrm{X}$ and $121-752 \mathrm{~A}-22 \mathrm{X}$

Holotype. Smear slide \#216010 deposited at the California Academy of Sciences (Pl. 4, Fig. 6).

Type locality. Eastern Indian Ocean, Sample 121-752A-22X-1, 55$57 \mathrm{~cm}$.

Age. Late Paleocene.

$$
\text { Stictodiscus bipolaris Fourtanier, n. sp. }
$$$$
\text { (PI. 1, Figs. 1-3; Pl. 5, Figs. 2-4) }
$$

Description. The valve outline is round with diameter ranging between 13 and $35 \mu \mathrm{m}$. The pore pattern of the valve has a bilateral symmetry. This symmetry is particularly obvious on larger valves where two or three parallel rows of pores located at the center of the valve serve as a starting point for radial rows of pores. The bilateral symmetry is not evident on smaller specimens (PI. 1, Fig. 1). The pores decrease in size from the center $(5-6$ in $10 \mu \mathrm{m})$ to the periphery $(9-11$ in $10 \mu \mathrm{m})$. The rows of pores are separated by ribs similar to those of other species of Stictodiscus.

Occurrence. Sporadic occurrence in the upper Paleocene of Site 752 (Cores 121-752A-18X to 121-752A-25X).

Holotype. Smear slide \#216011 deposited at the California Academy of Sciences (PI. 1, Fig. 3).

Type locality. Eastern Indian Ocean, Sample 121-752A-22X-3, 55$57 \mathrm{~cm}$.

Age. Late Paleocene.

\section{Species List and Taxonomic Notes}

This list includes all the taxa figured in Plates $1-5$ and considered in the occurrence chart. In addition, it includes some taxa observed sporadically in the material and not incorporated in the chart. For each taxa, reference to an illustration, either from this report (Pls. 1-5) and/or from other papers is given. Comments are included for several species.

Actinoptychus spp.

Anaulus fennerae n. sp. (description above; Pl. 4, Figs. 5-7; Pl. 5, Fig. 1).

Arachnoidiscus sp.

Coscinodiscus aff. undulosus Mann, 1907; Mann (1907), pl. 49, fig. 1.

Coscinodiscus sp. 1

Coscinodiscus spp.

Remarks. Common occurrence of large Coscinodiscus spp. is recorded at Site 752. These belong mostly to Coscinodiscus oculus iridis Ehr. group, Coscinodiscus marginatus Ehr. group, and Coscinodiscus radiatus Ehr. group.

Cosmiodiscus sp.

Fenestrella barbadensis Greville, 1863; Gombos (1984), pl. 6, figs. 1-4.

Hemiaulus inaequilateralus Gombos, 1977; Gombos (1977), pl. 20, figs. 5-7.

Remarks. Hemiaulus inaequilateralus is probably present at Site 752 (e.g., Pl. 3, Fig. 1); however, it is difficult to identify, especially when the valves are broken, and I could not determine precisely its stratigraphic range.

Hemiaulus incurvus Schibkova in Krotov and Schibkova, 1959 (Pl. 4, Fig. 11); Krotov and Schibkova (1959), p. 124, pl. 4, fig. 8; Gombos (1977), pl. 16, fig. 1-7.

Hemiaulus peripterus var, peripterus Fenner, 1984 (Pl. 3, Figs. 6, 7); Fenner (1984), pl. 1, figs. 8, 9.

Hemiaulus peripterus var. longispinus n. var. (described above; PI. 3, Figs. 5, 8, 9) 
Hemiaulus polycystinorum Ehrenberg, 1854 (Pl. 3, Fig. 4); Ehrenberg (1854), pl. 36, fig. 43 .

Hemiaulus polymorphus var. frigida Grunow, 1884; Mukhina (1976), pl. 1, fig. 7.

Hemiaulus sp. 1

Hemiaulus sp. 2 (PI. 3, Fig. 3).

Hemiaulus spp.

Hemiaulus? ciesielskii Fenner, 1991 (Pl. 4, Fig. 9); Fenner (1991), pl. 1, figs. 5,6 .

Hemiaulus? conicus Fenner, 1991 (PI. 4, Fig. 4); Fenner (1991), pl. 6, figs. 3-6.

Hemiaulus? velatus Fenner, 1991 (Pl. 4, Fig. 8); Fenner (1991), pl. 2, figs. 4-8, 11 .

Hemiaulus? sp. 3 (Pl. 4, Fig. 1).

Description. Only fragments of the apical elevations have been observed for this taxa. The long and straight apical elevation, terminated by an acute spine, has two rows of pores (6-7 pores in $10 \mu \mathrm{m})$.

Hyalodiscus aculeatus Paramonova, 1964 (P1. 1, Fig. 7); Paramonova (1964), figs. 1, 2.

Hyalodiscus scoticus (Kützing, 1844) Grunow, 1879 (PI. 1, Fig. 6); Mukhina (1976), pl. 2, fig. 6.

Hyalodiscus sp. 1 (Pl. 1, Fig. 9).

Hyalodiscus spp.

Melosira architecturalis s. ampl. Brun, 1892, in Schmidt et al. (18741959)

Remarks. Specimens observed at Site 752 do not correspond exactly to the original illustration of Melosira architecturalis Brun (Schmidt et al., 1874-1959, pl. 177, figs. 49-50).

Melosira concentrica Schmidt, 1887, in Schmidt et al., 1874-1959; Schmidt et al., 1874-1959, pl. 176, figs. 47-49; Fenner (1978), pl. 4, fig. 12.

Odontotropsis sp. Only rare fragments of Odontotropsis spp. were observed at Site 752 and were not determined at the specific level.

Paralia sulcata (Ehrenberg, 1838) Cleve, 1873

Pseudopodosira bella Posnova et Gleser in Gleser and Posnova, 1964 (P1. 1, Fig. 8); Gleser and Posnova (1964), table 1, fig. 2, text. fig. 1.

Pseudopodosira westii (W. Smith) Sheshukova-Poretzkaja et Gleser, 1964; Sheshukova-Poretzkaja and Gleser, 1964, table 1, figs. 4, 5.

Pseudopodosira sp. 1 (Pl. 1, Fig. 4)

Remarks. This species resembles Pseudopodosira mixta Posnova (Gleser and Posnova, 1964, fig. 2); however, it might be a different species as the central elevations are more irregularly arranged.

Pseudostictodiscus angulatus Grunow, 1882, in Schmidt et al., 18741959; Schmidt et al. (1874-1959), pl. 74, figs. 26-30.

Pseudotriceratium cheneveri (Meister, 1937) Gleser, 1975 (Pl. 2, Fig. 1); Gleser (1975), pl. 2, fig. 4.

Pterotheca evermanii Hanna, 1927; Fenner (1991), pl. 2, fig. 13.

Pterotheca spp.

Pyxilla gracilis Tempère et Forti in Forti, 1909 (Pl. 4, Fig. 10); Forti (1909), p. 24, pl. 2, fig. 5; Fenner (1985b), fig. 11.5.

Rhizosolenia cretacea Hajós et Stradner, 1975; Hajos and Stradner (1975), p. 929, pl. 7, fig. 7; pl. 31, figs. 4-6.

Rhizosolenia sp. aff. R. hebetata Bailey, 1856 (Pl. 4, Fig. 2).

Sceptroneis gemmata Grunow, 1886; Fenner (1985b), fig. 9.8.

Sceptroneis grunowii Anissimova, 1938; Gombos (1977), pl. 13, figs. 3, 4.

Sceptroneis spp.

Remarks. Various other species of the genus Sceptroneis are present at Site 752.

Skeletonema sp. (Pl. 1, Fig. 15)

Stellarima sp. 1 (PI. 1, Fig. 5)

Remarks. This species, relatively small in size (about $20-35 \mu \mathrm{m}$ in diameter), is characterized by a single elongated central labiate process.

Stellarima sp. 2

Remarks. Specimens grouped as Stellarima sp. 2 differ from Stellarima sp. 1 by larger size (up to $150 \mu \mathrm{m}$ in diameter) and by a greater number ( 2 to 7 ) of central labiate processes radially organized. The number of labiate processes might be related to the size of the frustule; therefore, Stellarima sp. 1 and Stellarima sp. 2 might be part of the same taxon. Stellarima sp. 1 and Stellarima sp. 2 may be equivalent to the specimens illustrated in Strelnikova (1974, pl. 12, figs. 1-7) as Coscinodiscus symbolophorus Grunow.
Stephanopyxis aff. schulzii Steinecke, 1935; Strelnikova (1974), pl. 4, figs. 1-3.

Stephanopyxis turris (Greville et Arnott, 1857) Ralfs in Pritchard, 1861. Stephanopyxis spp.

Stictodiscus bipolaris n. sp. (described above; Pl. 1, Figs. 1-3; P1. 5, Figs. 2-4).

Thalassiosiropsis sp.

Remarks. This species resembles the Cretaceous diatom Thalassiosiropsis wittiana (Pantocsek) Hasle (Hasle and Syvertsen, 1985).

Description. The valves are large ( 80 to $150 \mu \mathrm{m}$ diameter), the areolae (3-4 in $10 \mu \mathrm{m})$ are arranged in linear rows. A small process is present at the center.

Thalassiosira? sp. 1 (Pl. 1, Fig. 12)

Remarks. This species resembles the specimen illustrated in Schrader and Fenner (1976, pl. 33, fig. 6) as "genus and species indet."

Triceratium brachiatum Brightwell, 1856 (Pl. 2, Fig. 11); Fenner (1978), pl. 20, figs. 27-31.

Triceratium gombosii Fenner, 1991 (P1. 2, Fig. 8; P1. 5, Fig. 7); Fenner (1991), pl. 1, fig. 7, pl. 9, figs. 1, 2.

Remarks. This species is a synonym of Triceratium gracillium sensu Gombos (1977, pl. 38, fig. 5).

Triceratium gombosii var. A (Pl. 2, Figs. 6, 7; Pl. 5, Figs. 5, 6)

Description. The valves are small and triangular (usually between 15 and $25 \mu \mathrm{m}$ ). Slightly curved sulci separate the corners from the center. The central part of the valve is hyaline. Large pores are present near the sulci (ca. 6 in $10 \mu \mathrm{m}$ ), the corners are punctuated with smaller pores (ca. 10 in $10 \mu \mathrm{m}$ ). This taxa could be considered as a smaller form of Triceratium gombosii Fenner (1991). It differs from Triceratium gombosii by the lack of undulated sides; however, there is one ridge at the middle of each of the three sides.

Triceratium mirabile Jousé in Proshkina-Lavrenko, 1949 (Pl. 2, Figs. 4, 5); Jousé (1979), pl. 1, fig. 1; Fenner (1991), pl. 9, figs. 7-10.

Triceratium aff. mirabile Jousé

Triceratium orbiculatum Schibkova in Krotov and Schibkova, 1959 (P1. 2, Fig. 3); Krotov and Schikobva (1959), pl. 2, fig. 3.

Triceratium cf. tesselatum sensu Gombos, 1977 (P1. 2, Fig. 2)

Remarks. This species resembles Triceratium tesselatum Greville (cf. Williams, 1988, pl. 73, figs. 5, 6) by the shape of the valve but differs from it by the presence of a hyaline subrounded sulcus and by the complex microstructure of its areolae. It is equivalent to the specimen illustrated in Gombos (1977, pl. 37, figs. 1, 2) from the upper Paleocene of the South Atlantic Ocean.

Triceratium sp. 1 (Pl. 2, Fig. 13)

Triceratium sp. 3

Triceratium spp.

Trinacria aries Schmidt, 1886, in Schmidt et al., 1874-1959 (Pl. 2, Fig. 12); Schmidt et al. (1874-1959), pl. 96, figs. 14-17; Jousé (1982), pl. 7 , fig. 10, as Trinacria pileolus Ehrenberg.

Trinacria excavata Heiberg, 1863; Hustedt (1930), fig. 532.

Trinacria excavata Heiberg, 1863 f. tetragona Schmidt, 1890, in Schmidt et al., 1874-1959 (Pl. 2, fig. 9); Schmidt et al. (1874-1959), pl. 152, figs. 26-28; Gombos (1984), pl. 5, figs. 7-9.

Trinacria exsculpta (Heiberg, 1863) Hustedt, 1930; Mukhina (1976), pl. 2, fig. 7.

Trinacria pileolus (Ehrenberg) Grunow, 1884; Hustedt (1930), p. 885886, fig. 529; Gombos (1977), pl. 37, figs. 3, 4 .

Trinacria simulacrum Grove et Sturt, 1887; Gombos (1977), pls. 35-36.

Trochosira radiata Fenner, 1991 (PI. 1, fig. 14)

Trochosira spp. (PI. 1, Figs. 13, 16)

\section{ACKNOWLEDGMENTS}

I thank ODP France and INSU (Institut National des Sciences de l'Univers) for providing financial support for this study, and the USGS for providing space and facilities. Karen Hansen helped in sample preparation, and Robert Oscarson assisted in SEM studies. I thank John Barron for helpful discussions and constructive criticism of the manuscript. I am grateful to Juliane Fenner for communicating an early version of her ODP Leg 114 manuscript, and to David Harwood for discussion on taxonomy and for sending copies of Paleocene diatom literature. The manuscript 
benefited from the reviews of David Harwood, Andrew Gombos, and Juliane Fenner, and discussions with Nina Strelnikova.

\section{REFERENCES}

Benda, L., 1965. Diatomeen aus dem Eozän Norddeutschlands. Paläontol. Z., 39:165-187.

1972. The diatoms of the Moler Formation of Denmark (Lower Eocene): a preliminary report. Nova Hedwigia Beih., 39:251-266.

Berggren, W. A., Kent, D. V., and Flynn, J. J., 1985a. Jurassic to Paleogene: Part 2. Paleogene geochronology and chronostratigraphy. In Snelling, N. J. (Ed.), The Chronology of the Geological Record. Geol. Soc. London Mem., 10:141-195.

Berggren, W. A., Kent, D. V., and Van Couvering, J. A., 1985b. The Neogene: Part 2. Neogene geochronology and chronostratigraphy. In Snelling, N. J. (Ed.), The Chronology of the Geological Record. Geol. Soc. London Mem., 10:211-260.

de Prado, C. A., 1981. High-latitude Paleocene diatoms of the southern oceans [Master's thesis]. Northern Illinois Univ.

de Prado, C. A., and Ling, H. Y., 1981. Early to early middle Paleocene diatom zonation. Antarct. J. U. S., 14:124-125.

Dzinoridze, R. N., Jousé, A. P., Koroleva-Golikova, G. S., Kozlova, G. E., Nagaeva, M. G., Petrushevskaya, M. G., and Strelnikova, N. I., 1978. Diatom and radiolarian Cenozoic stratigraphy, Norwegian Basin; DSDP Leg 38. In Talwani, M., Udintsev, G., et al., Init. Repts., DSDP, Suppl. to vols. 38, 39, 40, and 41: Washington (U.S. Govt. Printing Office), 289-428.

Ehrenberg, C. G., 1854. Mikrogeologie das Erden und Felsen schaffende Wirken des unsichtbar kleinen selbständigen Lebens auf der Erde: Leipzig (Leopold Voss).

Fenner, J., 1978. Cenozoic diatom biostratigraphy of the equatorial and southern Atlantic Ocean. In Perch-Nielsen, K., Supko, P. R., et al., Init. Repts. DSDP, 39 (Suppl.): Washington (U.S. Govt. Printing Office), 491-624.

1984. Eocene-Oligocene planktic diatom stratigraphy in the low latitudes and the high southern latitudes. Micropaleontology, 30:319342.

1985a. Correction: Eocene-Oligocene planktic diatom stratigraphy in the low latitudes and in the high southern latitudes, Micropaleontology, vol. 30, no. 4, pp. 319-342. Micropaleontology, 31:197. 1985b. Late Cretaceous to Oligocene planktonic diatoms. In Bolli, H. M., Saunders, J. B., and Perch-Nielsen, K. (Eds.), Plankton Stratigraphy: Cambridge (Cambridge Univ. Press), 713-762.

1988. Occurrence of pre-Quaternary diatoms in Scandinavia reconsidered. Meyniana, 40:133-141.

1991. Taxonomy, stratigraphy, and paleooceanographic implications of Paleocene diatoms. In Ciesielski, P. F., Kristoffersen, Y., et al., Proc. ODP, Sci. Results, 114: College Station, TX (Ocean Drilling Program), 123-154.

Forti, A., 1909. Studi per una monografia del genere Pyxilla (Diatomee) e dei generi affini. Nuova Notarisia, 20:5-24.

Gleser, S. I., 1975. To the revision of the Genus Triceratium Ehr. sensu Hustedt, 1930 (Bacillariophyta). Acad. Sci. USSR, All-Union Botanical Soc., Botanical J., "NAUKA" (Leningrad Branch), 60:1304-1310, 3 pls.

1978. Znachenie kompleksov diatomykh i kremnevykh zhgutikovykh vodoroslei palaeogena zapadnoi Sibiri otdlya mezhregionalnykh korrelyatzii. Paleogen $i$ Neogen Sibiri (Paleontologiya $i$ Stratigrafiya) Mezhvedomstvennii Stratigrafischeskii Komitet SSSR, Sibirskaya Mezhvedomstvennaya Regionalya Kommissiya. Akad. Nauk SSSR, Sib. Otd., 49-67.

Gleser, S. I., and Posnova, A. N., 1964. Diatomeae Novae Marinae ex Eoceno Kazachstaniae occidentalis. Novitates Systematicae plantarum non vascularum. Academia Scientiarum URSS, Inst. Botanicum Nomine V.L. Komarovii, Moscow, Leningrad, NAUKA, 59-68.

Gombos, A. M., Jr., 1977. Paleogene and Neogene diatoms from the Falkland Plateau and Malvinas Outer Basin: Leg 36, Deep Sea Drilling Project. In Barker, P., Dalziel, I.W.D., et al., Init. Repts. DSDP, 36: Washington (U.S. Govt. Printing Office), 575-690.

1982. Early and middle Eocene diatom evolutionary events. Bacillaria, 5:225-242.
1984. Late Paleocene diatoms in the Cape Basin. In Hsü, K. J., LaBrecque, J. L., et al., Init. Repts. DSDP, 73: Washington (U.S. Govt. Printing Office), 495-512.

Grove, E., and Sturt, G., 1886-1887. On a fossil marine diatomaceous deposit from Oamaru, Otago, New Zealand. J. Quekett Microsc. Club Ser. 2, 2:321-330, 3:7-12, 3:63-78, 3:131-148.

Hajós, M., and Stradner, H., 1975. Late Cretaceous archaeomona-daceae, diatomaceae, and silicoflagellatae from the South Pacific Ocean, Deep Sea Drilling Project, Leg 29, Site 275. In Kennett, J. P., Houtz, R. E., et al., Init. Repts. DSDP, 29: Washington (U.S. Govt. Printing Office), 913-1009.

Harwood, D. M., 1988. Upper Cretaceous and lower Paleocene diatom and silicoflagellate biostratigraphy of Seymour Island, eastern Antarctic Peninsula. In Feldman, R., and Woodburne, M. O. (Eds.), Geology and Paleontology of Seymour Island. Geol. Soc. Am. Mem., 169:55-129.

Hasle, G. R., and Syvertsen, E. E., 1985. Thalassiosiropsis, a new diatom genus from the fossil records. Micropaleontology, 31:82-91.

Hustedt, F., 1930. Die Kieselalgen Deuchlands, Osterreichs und der Schweiz unter Berucksichtiging der übrigen Länder Europas sowie der angrenzenden Meeresgebiete (Vol. 1). In Rabenhorst L., Kryptogamen-Flora von Deutschland, Osterreich und der Schweiz: Koenigstein (O. Koeltz Science Publishers), reprint, 1977.

Jousé, A. P., 1979. Diatom biostratigraphic zones of the Eocene. Nova Hedwigia Beih., 64:427-445.

1982. Paleocene diatoms and silicoflagellates from the Pacific, Indian and Atlantic Ocean. Morskaya Mikropaleontol.: Moscow (NAUKA), 131-145.

Krotov, A. I., and Schibkova, K. G., 1959. Species novae diatomacearum e paleogeno montium uralensium (New species of diatoms from Paleogene deposits in the Urals). Botan. Mater. Otd. Sporovykh Rast., Botan. Inst., Akad. Nauk SSSR, 12:112-129.

1961. Kompleksy diatomovykh i kremnevykh zhgutikovykh vodoreslei $\mathrm{v}$ verkhnemelovykh paleogenovykh i neogenovykh otlozheniyakh vostokhnogo sklona Urala i Zauralya. Mater. Geol. Poleznym Iskopaemym Urala, 9:191-249.

Mann, A., 1907. Report on the diatoms of the Albatros voyages in the Pacific Ocean, 1888-1904. United States National Museum, Contributions from the United States National Herbarium, Vol. X, Part 5: Washington (U.S. Govt. Printing Office), 221-443.

Mukhina, V. P., 1974. The Paleocene diatom ooze in the eastern part of the Indian Ocean. Okeanologiya, 14:852-858. 1976. Species composition of the late Paleocene diatoms and silicoflagellates in the Indian Ocean. Micropaleontology, 22:151158.

Paramonova, N. V., 1964. Species novae diatomacearum ex Eoceni Sibiriae occidentalis. In Novitates Systematicae Plantarum non Vascularium: Moscow, Leningrad (NAUKA), 66-68.

Peirce, J., Weissel, J., et al., 1989. Proc. ODP, Init. Repts., 121: College Station, TX (Ocean Drilling Program).

Schmidt, A., Schmidt, M., Fricke, F., Heiden, H., Müller, O., and Hustedt, F. 1874-1959. Atlas der Diatomeen-Kunde. Leipzig (R. Reisland).

Schrader, H.-J., and Fenner, J., 1976. Norwegian Sea Cenozoic diatom biostratigraphy and taxonomy. In Talwani, M., Udintsev, G., et al., Init. Repts. DSDP, 38: Washington (U.S. Govt. Printing Office), 921-1098.

Sheshukova-Poretzkaja, V. S., and Gleser, Z. I., 1964. Diatomeae marinae novae e Paleoceno Ucrainiae. Novitates Systematicae plantarum non vascularum (Acad. Sci. URSS. Inst. Botanicum Nomine V.L. Komarovii) Moscow, Leningrad (NAUKA), 78-92.

Strelnikova, N. I., 1974. Diatoms of the Late Cretaceous: Moscow (NAUKA). [in Russian].

1987. Paleogene diatoms of the high latitudes of the northern and southern hemispheres [Doct. Sci. dissert.]. Univ. of Leningrad.

Williams, D. M., 1988. An illustrated catalogue of the type specimens in the Greville diatom herbarium. Bull. Br. Mus. Nat. Hist. (Bot.), 148.

Date of initial receipt: 21 March 1990

Date of acceptance: 4 October 1990

Ms 121B-136 

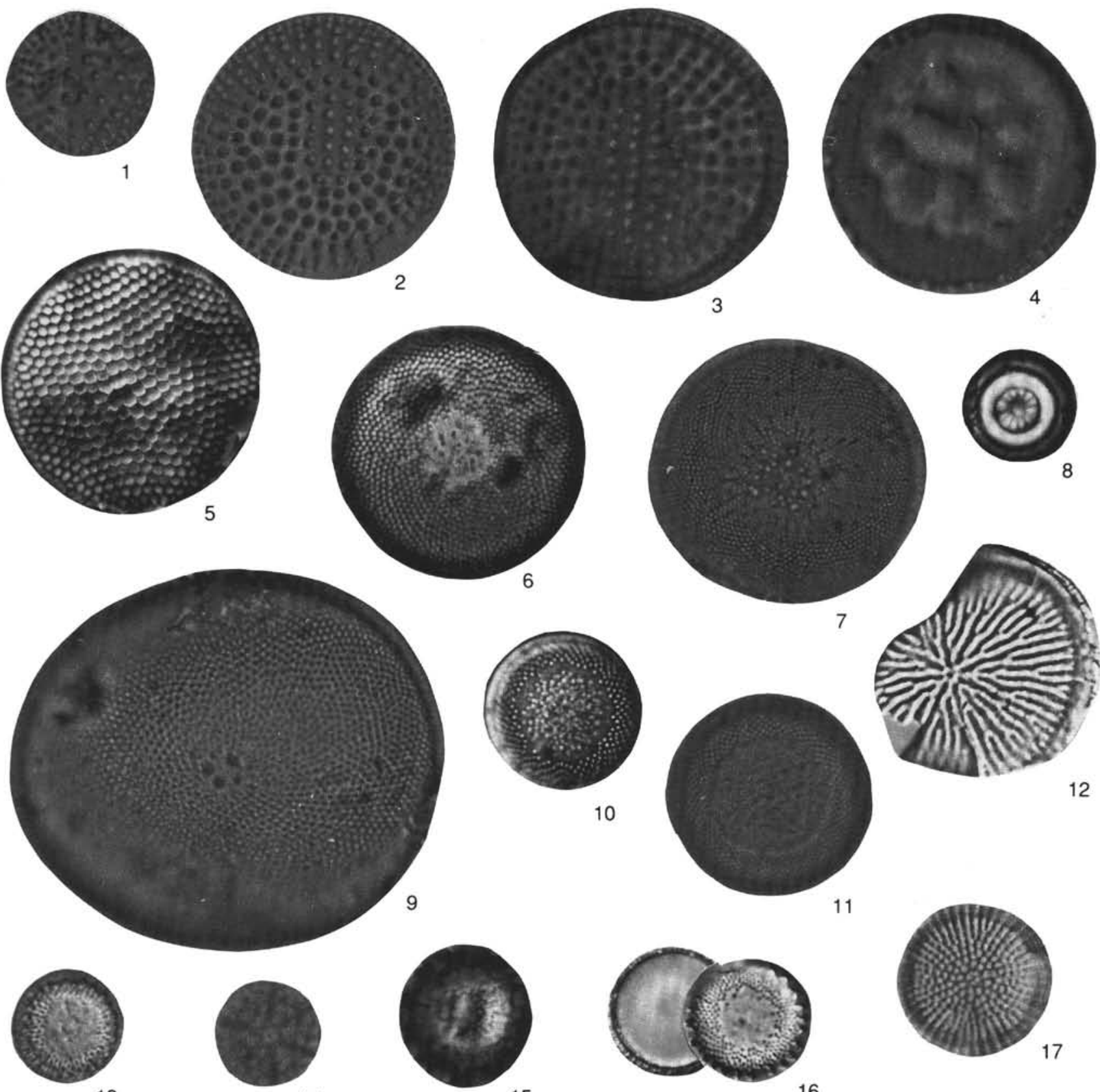

13

14

15
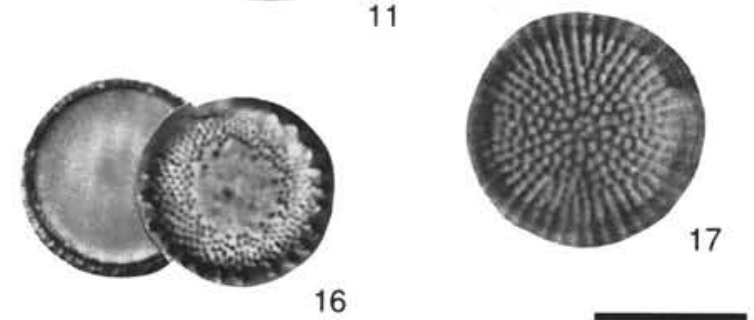

Plate 1. (All figures 1500x; scale bar $=10 \mu \mathrm{m}$ ). 1. Stictodiscus bipolaris n. sp., Sample 121-752A-21X-1, $55-57 \mathrm{~cm}$. 2. Stictodiscus bipolaris n. sp., Sample 121-752A-22X-3, 55-57. 3. Stictodiscus bipolaris, n. sp. (holotype), Sample 121-752A-22X-3, 55-57 cm. 4. Pseudopodosira sp. 1, Sample 121-752A-22X-1, 55-57 cm. 5. Stellarima sp. 1, Sample 121-752A-26X-1, 55-57 cm. 6. Hyalodiscus scoticus (Kützing) Grunow, Sample 121-752A-24X-1, 55-57 cm. 7. Hyalodiscus aculeatus Paramonova, Sample 121-752A-25X-1, 53-55 cm. 8. Pseudopodosira bella Posnova et Gleser, Sample 121-752A-24X-1, 55-57 cm. 9. Hyalodiscus sp. 1, Sample 121-752A-25X-1, 53-55 cm. 10, 11. Hyalodiscus spp., Sample 121-752A-21X-1, 55-57 cm. 12. Thalassiosira? sp. 1, Sample 121-752A-24X-1, 55-57 cm. 13. Trochosira sp., Sample 121-752A-17X-3, 55-57 cm. 14. Trochosira radiata Fenner, Sample 121-752A-22X-3, 55-57 cm. 15. Skeletonema sp., Sample 121-752A-26X-1, 55-57 cm. 16. Trochosira sp., Sample 121-752A-21X-1, 55-57 cm (low and high focus). 17. Trochosira? sp., Sample 121-752A-17X-3, 55-57 cm. 


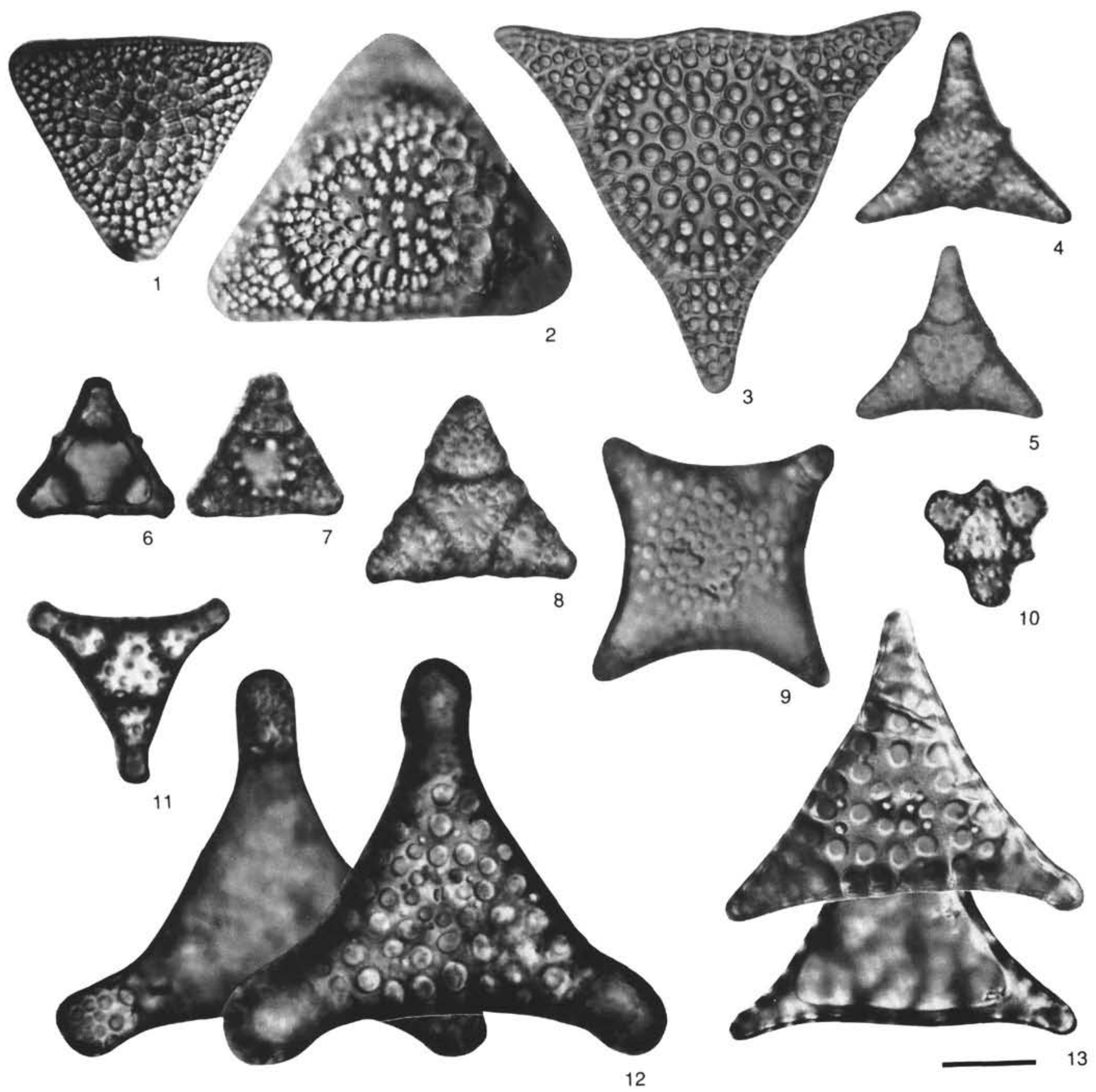

Plate 2. (All figures 1500x; scale bar $=10 \mu \mathrm{m}$ ). 1. Triceratium cheneveri (Meister) Gleser, Sample 121-752A-21X-1, 55-57 cm. 2. Triceratium cf. tesselatum Greville sensu Gombos 1976, Sample 121-752A-26X-1, 55-57 cm. 3. Triceratium orbiculatum Schibkova, Sample 121-752A-18-CC. 4. Triceratium mirabile Jousé, Sample 121-752A-24X-1, 55-57 cm. 5. Triceratium mirabile Jousé, Sample 121-752A-26X-3, 55-57 cm. 6, 7. Triceratium gombosii var. A, Sample 121-752A-26X-1, 55-57 cm. 8. Triceratium gombosii Fenner, Sample 121-752A-22X-3, 55-57 cm. 9. Trinacria excavata f. tetragona Schmidt, Sample 121-752A-18X-2, 53-55 cm. 10. Triceratium sp., Sample 121-752A-26X-1, 55-57 cm. 11. Triceratium brachiatum Brightwell, Sample 121-752A-24X-1, $55-57 \mathrm{~cm}$. 12. Trinacria aries Schmidt (high and low focus), Sample 121-752A-24X-1, 55-57 cm. 13. Triceratium sp. 1 (high and low focus), Sample $121-752 \mathrm{~A}-14 \mathrm{X}-3,55-57 \mathrm{~cm}$. 

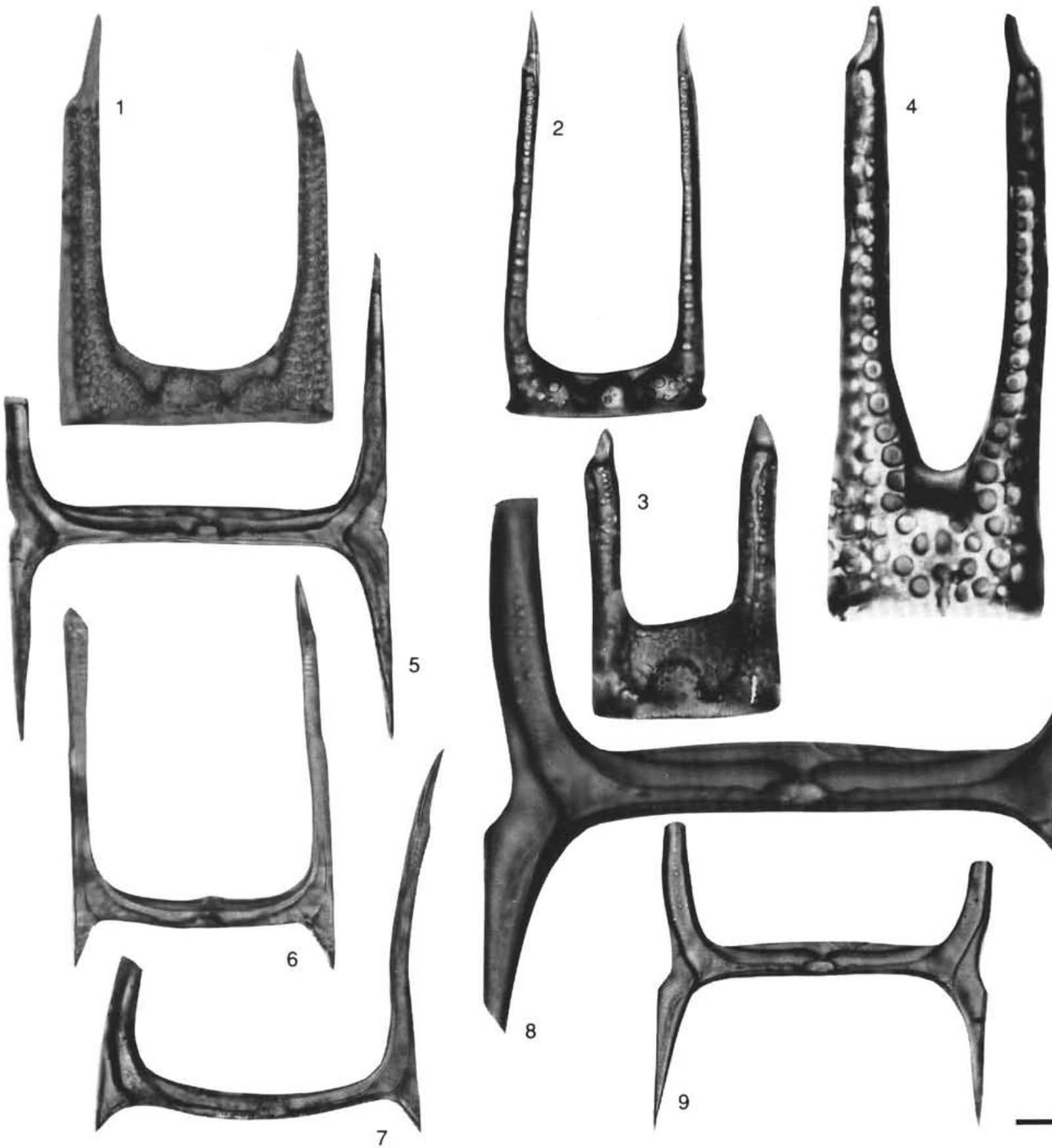

Plate 3. (All figures $800 \times$, except Figs. 4 and 8 are $1500 \times$; scale bar $=19 \mu \mathrm{m}$ for all figures, except Figs. 4 and $8=10 \mu \mathrm{m}$ ). 1. Hemiaulus inaequilateralus? Gombos, Sample 121-752A-22X-1, 55-57 cm. 2. Hemiaulus sp., Sample 121-752A-18X-2,53-55 cm. 3. Hemiaulus sp. 2, Sample 121-752A-17X-1, 55-57 cm. 4. Hemiaulus polycystinorum Ehrenberg, Sample 121-752A-21X-1, 55-57 cm. 5. Hemiaulus peripterus var. longispinus $\mathrm{n}$. var., Sample 121-752A-17X-3, 55-57 cm. 6. Hemiaulus peripterus var. peripterus Fenner, Sample 121-752A-29X-3, 55-57 cm. 7. Hemiaulus peripterus var. peripterus Fenner, Sample 121-752A-29X-3, $55-57 \mathrm{~cm}$. 8, 9. Hemiaulus peripterus var. longispinus $\mathrm{n}$. var. (holotype), same specimen at different enlargements, Sample 121-752A-17X-3, 55-57 cm. 


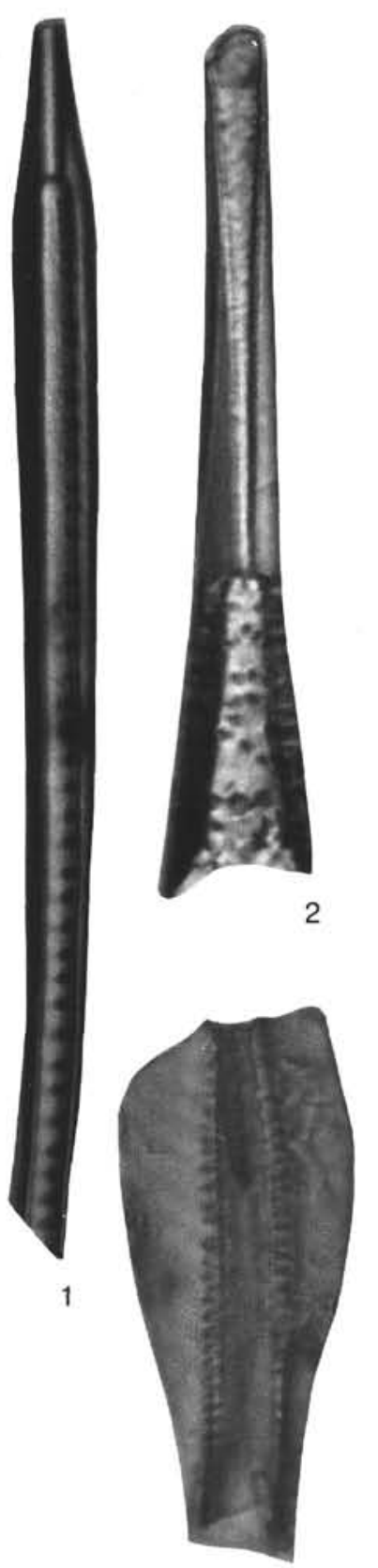

8

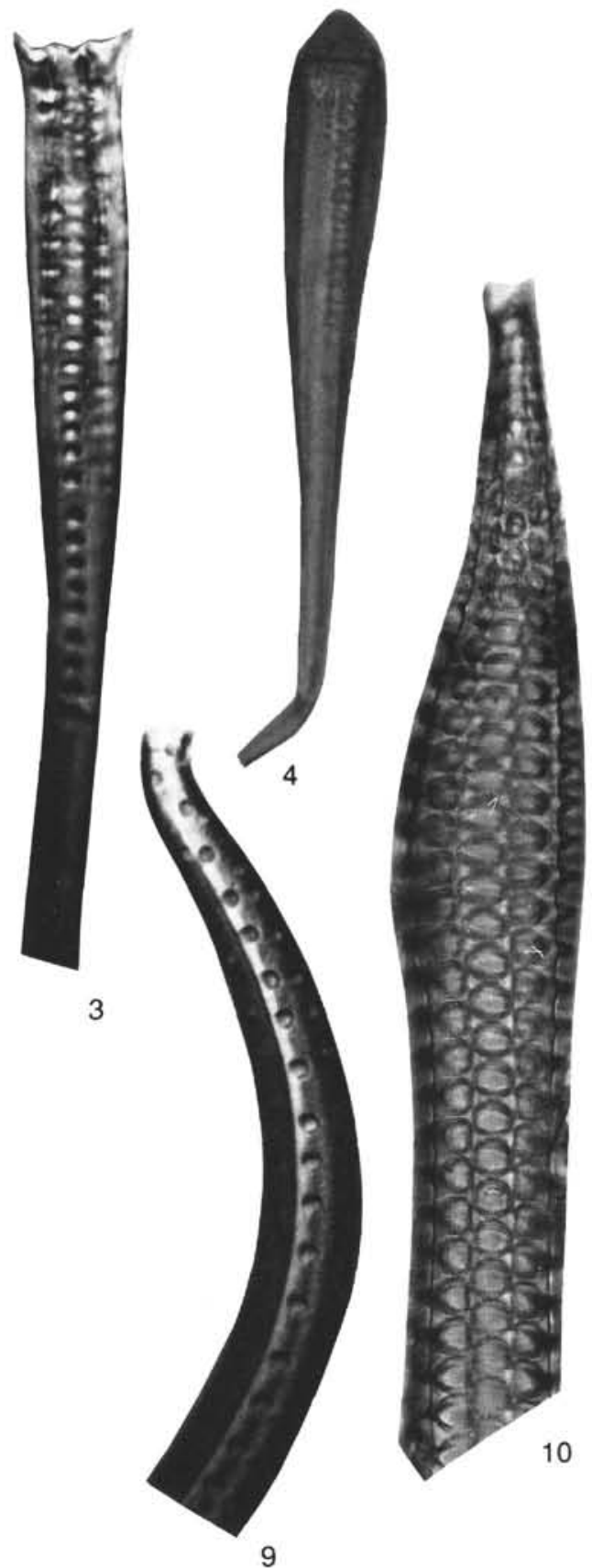

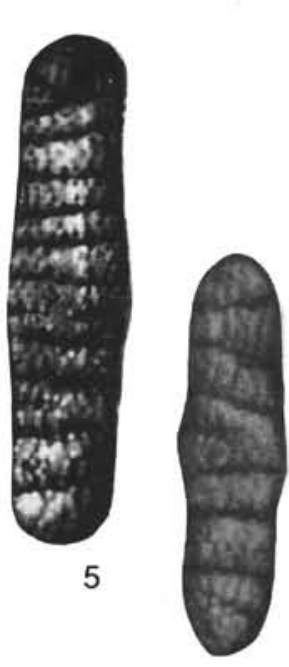

$$
6
$$
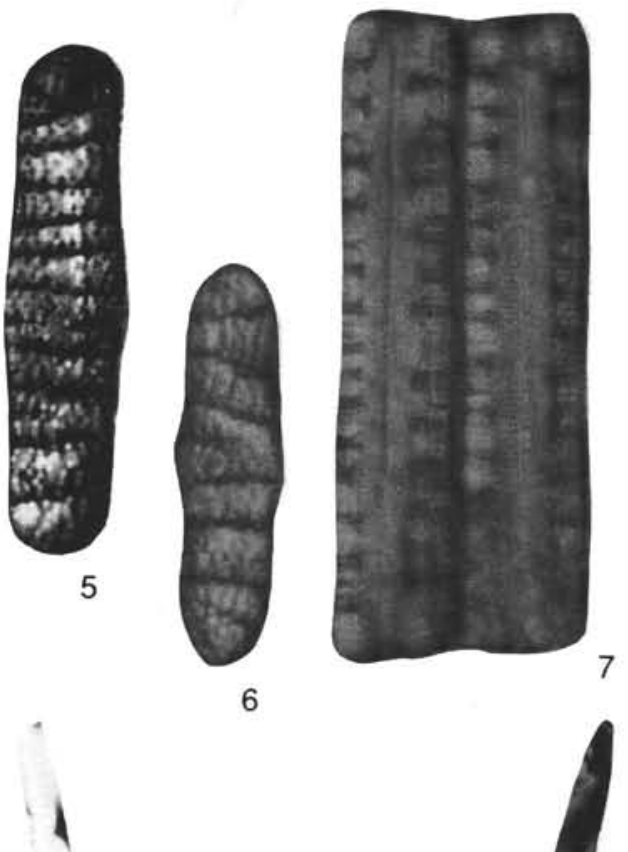

7

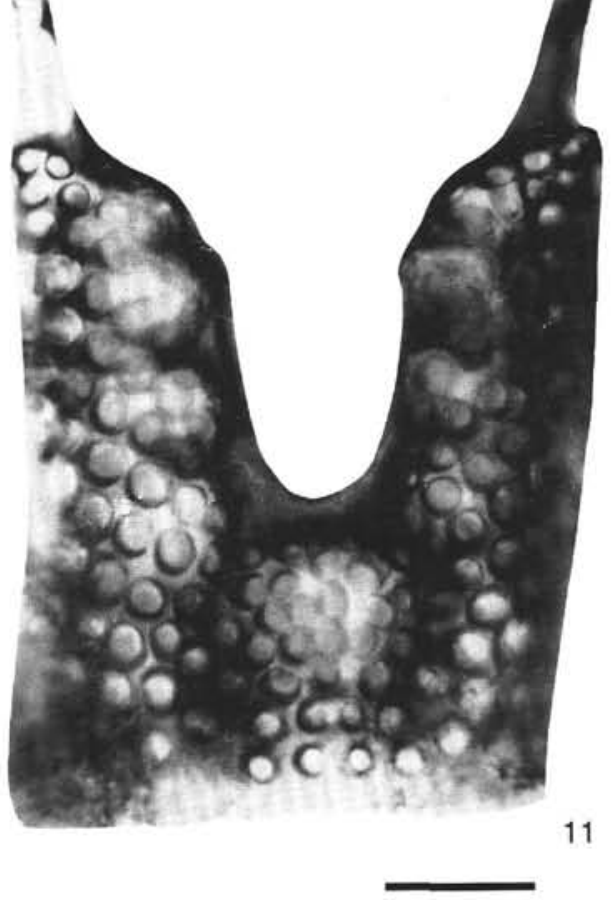

11

Plate 4. (All figures $1500 x$, except Fig. 10 is $800 x$; scale bar $=10 \mu \mathrm{m}$ for all figures, except Fig. $10=19 \mathrm{~mm}$ ). 1. Hemiaulus? sp. 3, Sample 121-752A-16X-5, $7-9 \mathrm{~cm}$. 2. Rhizosolenia sp. aff. $R$. hebetata Bailey, Sample 121-752A-24X-1, 55-57 cm. 3. Hemiaulus? velatus Fenner, Sample 121-752A-26X-1, 55-57 cm. 4. Hemiaulus? conicus Fenner, Sample 121-752A-25X-1, 53-55 cm. 5-7. Anaulus fennerae n. sp.; (5) holotype, Sample 121-752A-21-CC, (6) valvar view, Sample 121-752A-22X-1, 55-57 cm, (7) connective view, Sample 121-752A-21-CC. 8. Hemiaulus? velatus Fenner, Sample 121-752A-25X-3, 53-55 cm. 9. Hemiaulus? ciesielskii Fenner, Sample 121-752A-21X-1, 55-57 cm. 10. Pyxilla gracilis Tempère et Forti, Sample 121-752A-17X-1, 55-57 cm. 11. Hemiaulus incurvus Schibkova, Sample 121-752A-21X-CC. 

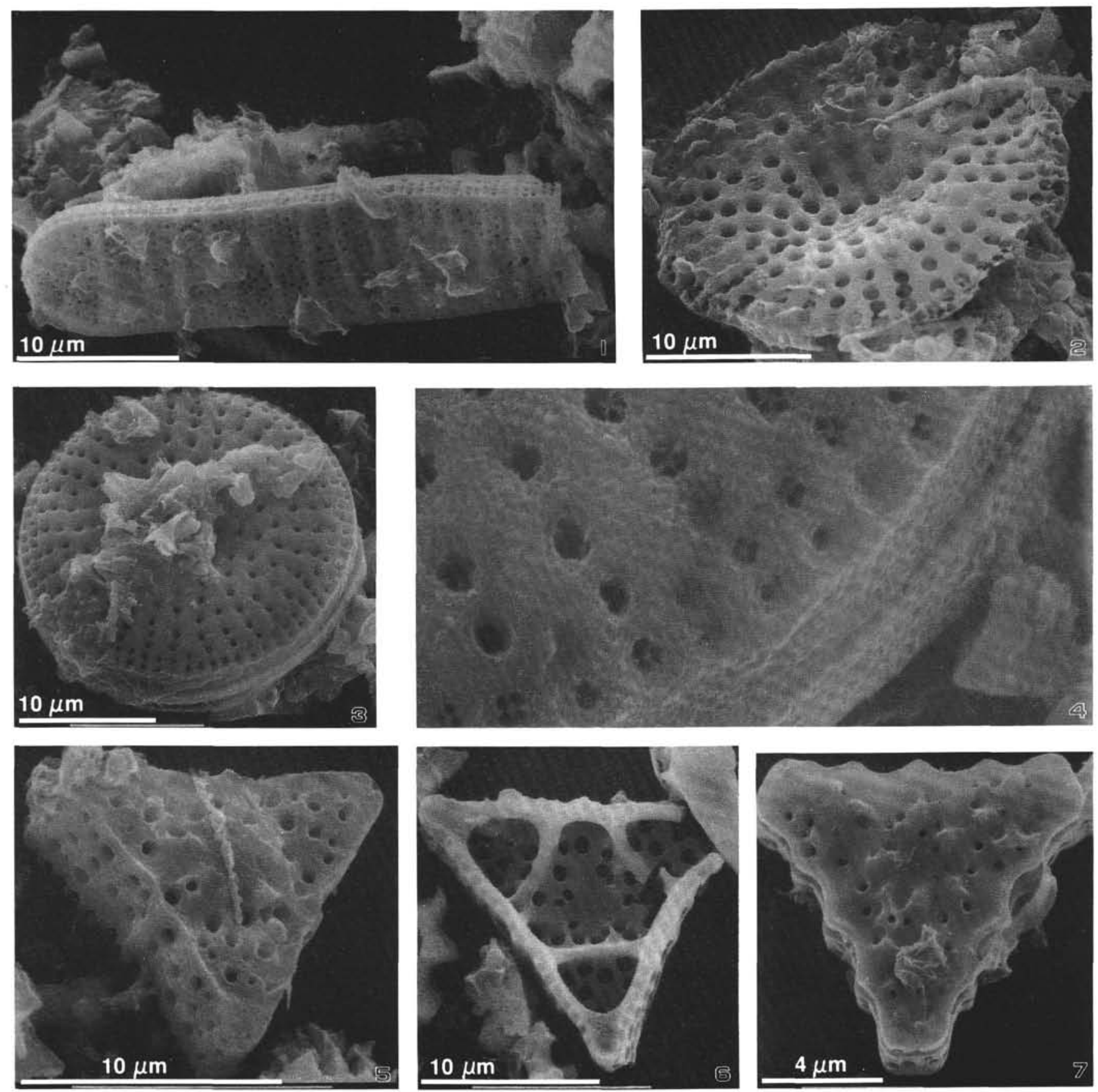

Plate 5. SEM photographs of diatoms from Hole 752A. 1. Anaulus fennerae n. sp., Sample 121-752A-21X-CC. 2-4. Stictodiscus bipolaris n. sp., Sample 121-752A-22X-3, 55-57 cm. 5, 6. Triceratium gombosii Fenner var. A, Sample 121-752A-29X-3, 55-57 cm; (5) external view, (6) internal view. 7. Triceratium gombosii Fenner, Sample 121-752A-29X-3, 55-57 cm. 